University of Wollongong

Research Online

Faculty of Science, Medicine and Health -

Papers: part A

Faculty of Science, Medicine and Health

$1-1-2012$

\title{
Nanocavitation in carbon black filled styrene-butadiene rubber under tension detected by real time small angle X-ray scattering
}

\author{
Huan Zhang \\ Espci Paristech-Cnrs-Upmc \\ Arthur K. Scholz \\ University of California \\ Jordan Crevoisier \\ Espci Paristech-Cnrs-Upmc \\ Fabien Vion-Loisel \\ Cerl Ladoux \\ Gilles Besnard \\ Ens-Cachan
}

See next page for additional authors

Follow this and additional works at: https://ro.uow.edu.au/smhpapers

\section{Recommended Citation}

Zhang, Huan; Scholz, Arthur K.; Crevoisier, Jordan; Vion-Loisel, Fabien; Besnard, Gilles; Hexemer, Alexander; Brown, Hugh R.; Kramer, Edward; and Creton, Costantino, "Nanocavitation in carbon black filled styrene-butadiene rubber under tension detected by real time small angle X-ray scattering" (2012). Faculty of Science, Medicine and Health - Papers: part A. 29.

https://ro.uow.edu.au/smhpapers/29

Research Online is the open access institutional repository for the University of Wollongong. For further information contact the UOW Library: research-pubs@uow.edu.au 


\title{
Nanocavitation in carbon black filled styrene-butadiene rubber under tension detected by real time small angle $X$-ray scattering
}

\begin{abstract}
"Nanocavitation was detected for the first time in carbon black filled styrene-butadiene rubber (CB-SBR) under uniaxial loading by real time small-angle X-ray scattering (SAXS) using synchrotron X-ray radiation. A three phase model was developed to calculate the void volume fraction from the scattering invariant $Q$ determined from the observed SAXS patterns. The normalized scattering invariant $Q / Q(0)$, where $Q(0)$ is the invariant before deformation, greatly increased above a critical extension ratio lambda(onset) which we attribute to the formation of nanovoids. Analysis of the 2D scattering patterns show that voids formed are $20-40 \mathrm{~nm}$ in size and elongated along the tensile direction. Cavities formed beyond lambda(onset) are smaller as lambda increases. Results from the scattering experiments are strongly supported by macroscopic volume change measurements on the samples under similar uniaxial strain. A nearly constant nanocavitation stress sigma(onset) (25 MPa) was observed when the filler volume fraction phi(CB) was larger than $14 \%$. This value is much higher than that predicted based on the elastic instability of small voids in an unfilled elastomer and shows only a weak dependence on the cross-linking density $\mathrm{v}(\mathrm{C})$ in heavily cross-linked samples. An energy based cavitation criterion stressing the importance of confined domains between particles or clusters of particles was adopted and found to be consistent with the observed results. The nanocavities are thought to alter the local stress state and promote local shear motion of filler particles."
\end{abstract}

\section{Keywords}

ray, $x$, angle, small, time, real, detected, scattering, tension, nanocavitation, under, rubber, butadiene, styrene, filled, black, carbon

\section{Publication Details}

Zhang, H., Scholz, A. K., Crevoisier, J., Vion-Loisel, F., Besnard, G., Hexemer, A., Brown, H. R., Kramer, E. \& Creton, C. (2012). Nanocavitation in Carbon Black Filled Styrene-Butadiene Rubber under Tension Detected by Real Time Small Angle X-ray Scattering. Macromolecules, 45 (3), 1529-1543.

\section{Authors}

Huan Zhang, Arthur K. Scholz, Jordan Crevoisier, Fabien Vion-Loisel, Gilles Besnard, Alexander Hexemer, Hugh R. Brown, Edward Kramer, and Costantino Creton 


\title{
Nanocavitation in Carbon Black Filled Styrene-Butadiene Rubber under Tension Detected by Real Time Small Angle X-ray Scattering
}

\author{
Huan Zhang, ${ }^{\dagger}$ Arthur K. Scholz, ${ }^{\ddagger} \S$ Jordan de Crevoisier, ${ }^{\dagger, \mathbb{I L}}$ Fabien Vion-Loisel," Gilles Besnard, ${ }^{\text {II }}$ \\ Alexander Hexemer, $\triangle^{\triangle}$ Hugh R. Brown, ${ }^{\#}$ Edward J. Kramer, ${ }^{*}, \$,, \perp$ and Costantino Creton ${ }^{* \dagger}$ \\ ${ }^{\dagger}$ Laboratory of Soft Matter Science and Engineering, ESPCI Paristech-CNRS-UPMC, 10 rue Vauquelin, 75005 Paris, France \\ ${ }^{\ddagger}$ Materials Research Laboratory, University of California, Santa Barbara, California 93106, United States \\ ${ }^{\S}$ Department of Materials, University of California, Santa Barbara, California 93106, United States \\ ${ }^{\perp}$ Department of Chemical Engineering, University of California, Santa Barbara, California 93106, United States \\ "Michelin, CERL Ladoux, F-63040 Clermont Ferrand, France

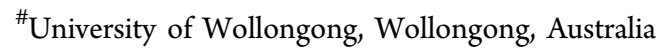 \\ II LMT-Cachan, ENS-Cachan, 61 avenue du président Wilson, F-94230 Cachan, France \\ $\triangle$ Advanced Light Source, Lawrence Berkeley National Laboratory, 1 Cyclotron Road, Berkeley, California 94720, United States
}

Supporting Information

ABSTRACT: Nanocavitation was detected for the first time in carbon black filled styrene-butadiene rubber (CB-SBR) under uniaxial loading by real time small-angle $\mathrm{X}$-ray scattering (SAXS) using synchrotron X-ray radiation. A three phase model was developed to calculate the void volume fraction from the scattering invariant $Q$ determined from the observed SAXS patterns. The normalized scattering invariant $Q / Q_{0}$, where $Q_{0}$ is the invariant before deformation, greatly increased above a critical extension ratio $\lambda_{\text {onset }}$ which we attribute to the formation of nanovoids. Analysis of the $2 \mathrm{D}$ scattering patterns show that voids formed are $20-40 \mathrm{~nm}$ in size and elongated along the tensile direction. Cavities formed beyond $\lambda_{\text {onset }}$ are smaller as $\lambda$ increases. Results from the scattering experiments are strongly supported by macroscopic volume change measurements on the samples under similar uniaxial strain. A nearly constant nanocavitation stress $\sigma_{\text {onset }}(25 \mathrm{MPa})$ was observed when the filler volume fraction $\phi_{\mathrm{CB}}$ was larger than $14 \%$. This value is much higher than that predicted based on the elastic instability of small voids in an unfilled elastomer and shows only a weak dependence on the cross-linking density $\nu_{\mathrm{C}}$ in heavily cross-linked samples. An energy based cavitation criterion stressing the importance of confined domains between particles or clusters of particles was adopted and found to be consistent with the observed results. The nanocavities are thought to alter the local stress state and promote local shear motion of filler particles.

\section{INTRODUCTION}

Elastomers are highly deformable and nearly incompressible materials that find a variety of applications where high mechanical strength needs to be combined with the accommodation of large strains. Yet to obtain these remarkable properties noncrystallizing elastomers need to be filled with nanoparticles, generally carbon black (CB) or silica. The presence of such nanoparticles introduces significant heterogeneities in the material structure and complexity in the mechanical properties of the materials. As a result the investigation of the connection between material morphology and structure, and mechanical properties has been the focus of research for decades. ${ }^{1-4}$ Although the presence of nanoparticles has important effects on the dissipative properties at small strain, the focus of the present study is on the failure mechanisms of elastomers at large strains, mechanisms that are particularly relevant to understanding macroscopic fracture processes.

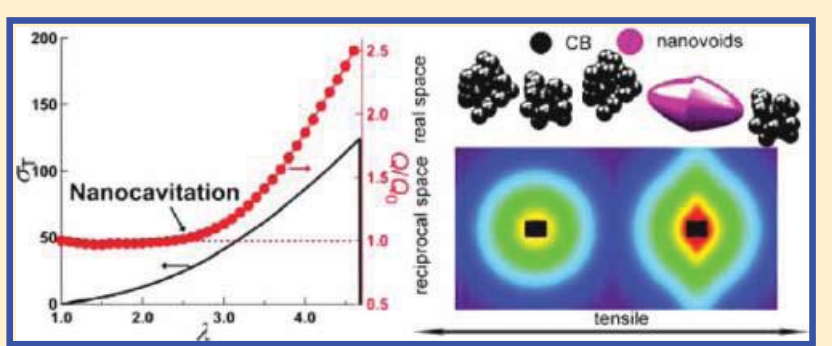

Because the bulk modulus of elastomers greatly exceeds their shear and Young's moduli, they can store large amounts of volumetric strain energy at relatively low strains if they are loaded in a confined geometry where tensile stresses are triaxial. If triaxial tensile stresses are present over a macroscopic volume, optically visible vacuoles typically appear and grow in the bulk above a critical applied stress. Such a failure process is often termed "cavitation". These cavitation phenomena have been studied in the past essentially with two experimental methods: direct optical visualization of cavities either in situ ${ }^{5-9}$ or post mortem, ${ }^{10}$ and measurements of volume increase of the filled elastomers during stretching using a gas or liquid dilatometer. ${ }^{8,9,11,12}$ Such volume change experiments could be of

Received: October 22, 2011

Revised: January 5, 2012

Published: January 27, 2012 
course carried out equally well on unfilled elastomers or on filled elastomers with variable particle sizes and any positive change in volume was interpreted as a sign of cavitation or decohesion. Only rubbers filled with large volume fractions of micrometer size particles showed a significant volume change in uniaxial tension. ${ }^{9}$ Criteria for the growth of such cavities (either by deformation or by fracture) under a hydrostatic tensile stress were proposed. ${ }^{10,13-17}$ Yet individual cavities could only be detected with an optical microscope above a size of a few micrometers. The question of the nucleation of such large cavities remains therefore very much an open question.

In the case of elastomers filled with nanoparticles, in addition to the macroscopic cavities discussed above, it is reasonable to think that the presence of randomly dispersed nanoparticles and particle aggregates can create nanosized rubber domains between filler aggregates, domains that can be loaded in triaxial tension even if the macroscopic sample is deformed uniaxially. Under high tensile stresses such confined domains are prone to create nanocavities which could then act as precursors for macroscopic cracks. Yet the formation of nanovoids in filled rubbers in uniaxial tension has never been directly demonstrated. Experiments by LeCam et al. ${ }^{18,19}$ and by Starkova and Aniskevich ${ }^{20}$ showed indeed an increase in macroscopic volume under uniaxial tension but no direct evidence has been observed to relate it with nanovoids. A direct relationship between voids and volume changes has been convincingly demonstrated for larger particles ${ }^{8,9,11}$ (tens of micrometers), very different from the materials of the present study. Ramier et al. ${ }^{21}$ studied silica-filled SBR by a combination of techniques (dilatometry, light scattering, SAXS and USAXS) and only detected large cavities by small angle light scattering in a thin rubber film and qualitatively associated them with the change in macroscopic volume of the material but did not detect any nanocavities by USAXS and SAXS. In summary, no direct evidence has been observed to relate the positive changes in volume measured during uniaxial extension with the presence of nanovoids, their size or size distribution. Yet recent observations of crack propagation in filled rubbers reveal fibrillar structures at the micrometer scale at the crack tip ${ }^{22,23}$ that suggest the genesis and development of submicrometer cavities ahead of the crack front that should be detectable by SAXS.

Thanks to the development of high flux synchrotron X-ray facilities, small-angle X-ray scattering (SAXS) has been used as a powerful tool for real time detection of changes in structure under strain. Many studies have focused on rubber toughened plastics $^{24,25}$ and on semicrystalline polymers. ${ }^{26-28}$ However several studies have also been published for filled elastomers under uniaxial extension. ${ }^{21,29-36}$ Most of them focused on the change of anisotropy of the system, ${ }^{29,30}$ the changes in fractal dimension of the filler network ${ }^{31-33}$ and the rearrangement of filler in real space under external loading. ${ }^{34-36}$ Again, no nanocavitation was discovered in those filled systems.

The objective of the current work is to develop a methodology to quantitatively detect nanovoids in a noncrystallizing filled rubber and to investigate the effect of material parameters and loading parameters on such a process. All experiments were performed on a series of model filled elastomers made from an identical random copolymer of styrene-butadiene (SBR), vulcanized with a sulfur-based chemistry and filled with various proportions of the same type of carbon black. Since in carbon black filled elastomers the use of optical methods is ruled out by the absorption of light by the carbon black particles, the nanovoid formation was detected by SAXS experiments performed at synchrotron X-ray facilities during tensile tests. Our main analytical tool was the analysis of $2 \mathrm{D}$ scattering patterns and in particular we exploited the scattering invariant following a method proposed as far back as 1950 by Debye ${ }^{37}$ and developed to investigate crazes in glassy polymers by Paredes, Fischer, ${ }^{38,39}$ and Brown and Kramer. ${ }^{40-42}$ To adapt the method to our system, a new three phase model was developed to calculate the volume fraction of nanovoids in the presence of both elastomer and filler from the 2D scattering data obtained during uniaxial extension experiments. The shape and size of the voids was then obtained from the detailed analysis of the $2 \mathrm{D}$ scattering pattern.

\section{EXPERIMENTAL SECTION}

1. Sample Preparation. Michelin prepared various samples of CB filled SBR rubber for this study. The SBR $\left(M_{\mathrm{n}}=120 \mathrm{~kg} / \mathrm{mol}\right.$, $M_{\mathrm{w}} / M_{\mathrm{n}}=1.94, T_{\mathrm{g}}=-48{ }^{\circ} \mathrm{C}$ and density $\left.d_{\mathrm{SBR}}=0.94 \mathrm{~g} / \mathrm{cm}^{3}\right)$ used was a random copolymer with $0.15 \mathrm{~mol}$ fraction styrene. The filler was carbon black (reference N347) with surface area (cetyltrimethylammonium bromide adsorption) of $87 \pm 5 \mathrm{~m}^{2} / \mathrm{g}$ and structure (dibutylpthlate adsorption) of $124 \pm$ $5 \mathrm{~mL} / 100 \mathrm{~g}$ and density $d_{\mathrm{CB}}=1.8 \mathrm{~g} / \mathrm{cm}^{3}$. The amount of CB loaded in the SBR matrix was between 5 and $60 \mathrm{phr}$ (parts of weight per hundred parts of rubber) which covered a range of volume fractions from $2.4 \%$ to $23.9 \%$ respectively. The crosslinking system was based on sulfur and $n$-cyclohexy12-benzothiazyl-sulfenamide (CBS). The antioxidant $\mathrm{N}$-(1,3dimethylbutyl)- $N$ '-phenyl-p-phenylenediamine 6PPD (1.9 phr), the vulcanization activators stearic acid (2 phr) and zinc oxide $(\mathrm{ZnO}, 2.5 \mathrm{phr})$ were the same for all the samples. Elastomer and filler were mixed in a chamber at $50{ }^{\circ} \mathrm{C}$ first. Then in the second step, the CBS accelerators and sulfur were added using two-roll mills. Samples were cured at $150{ }^{\circ} \mathrm{C}$ for different times. The important parameters of the filled SBR samples are summarized in Table 1 . The samples are designated as

Table 1. Characteristics of the Samples

\begin{tabular}{ccccccc} 
label & $\begin{array}{c}\text { SBR } \\
(\mathrm{phr})\end{array}$ & $\begin{array}{c}\mathrm{CB} \\
(\mathrm{vol} \%)\end{array}$ & $\begin{array}{c}\text { sulfur } \\
(\mathrm{phr})\end{array}$ & $\begin{array}{c}\mathrm{CBS} \\
(\mathrm{phr})\end{array}$ & $\begin{array}{c}\nu_{\mathrm{c}}\left(10^{-5}\right. \\
\left.\mathrm{mol} / \mathrm{cm}^{3}\right)\end{array}$ & $\begin{array}{c}\text { curing } \\
\text { time } \\
(\mathrm{min})\end{array}$ \\
\hline 2CB_8NC & 100 & 2.3 & 1.6 & 1.6 & 6.5 & 35 \\
14CB_8NC & 100 & 13.5 & 1.6 & 1.6 & 8.2 & 23 \\
17CB_8NC & 100 & 17.3 & 1.6 & 1.6 & 7.4 & 20 \\
21CB_8NC & 100 & 20.7 & 1.6 & 1.6 & 8.3 & 20 \\
24CB_8NC & 100 & 23.9 & 1.6 & 1.6 & 7.7 & 15 \\
17CB_0NC & 100 & 17.3 & 1.6 & 1.6 & $/$ & $/$ \\
17CB_4NC & 100 & 17.3 & 1 & 1 & 3.6 & 41 \\
17CB_11NC & 100 & 17.3 & 2.3 & 2.3 & 10.6 & 18 \\
17CB_15NC & 100 & 17.3 & 4.7 & 4.7 & 15.5 & 15 \\
\hline
\end{tabular}

$m \mathrm{CB} \_n \mathrm{NC}$ where $m$ is the volume fraction of carbon black and $n$ is the nominal molar density of cross-links in $10^{-5} \mathrm{~mol} / \mathrm{cm}^{3}$. The cross-linking density $\nu_{\mathrm{c}}$ was obtained by swelling experiments analyzed using the Flory-Rehner equation ${ }^{43}$ assuming that the filler cannot swell.

Dog-bone shaped samples were cut from rubber sheets for mechanical testing with a gauge length of $50 \mathrm{~mm} \times 4 \mathrm{~mm}$ (width) $\times 2 \mathrm{~mm}$ (thickness). All samples were kept at $-18{ }^{\circ} \mathrm{C}$ to prevent aging until shipping to the beamline.

2. Small Angle X-ray Scattering Measurement. The SAXS measurements were performed at the synchrotron beamline 7.3.3 at the Advanced Light Source, Lawrence 
Berkeley National Laboratory, Berkeley, CA. The energy of the $\mathrm{X}$-ray radiation was $10 \mathrm{keV}$, resulting in an X-ray wavelength $\lambda_{\text {wave }}$ of $0.127 \mathrm{~nm}$. The incident beam intensity IC1 (counts per second) and the transmitted beam intensity IC2 (counts per second) were measured by an ion chamber before the samples and a pin diode in the beam stop, respectively. Two dimensional scattering patterns were recorded by a Pilatus $1 \mathrm{M}$ detector (pixel size $0.172 \mu \mathrm{m}, 1043$ pixels $\times 981$ pixels). The sample to detector distance was roughly $4 \mathrm{~m}$ and was calibrated using an Ag-behenate standard sample. The size of the beam spot is $1 \mathrm{~mm}$ (horizontal) $\times 0.7 \mathrm{~mm}$ (vertical). The scattered intensity could be measured down to a minimum value of the scattering vector $q\left(q=2 \pi \sin (2 \theta) / \lambda_{\text {wave, }}\right.$ where $2 \theta$ is the scattering angle) of $0.025 \mathrm{~nm}^{-1}$.

Because IC 1 and IC2 were measured by two different devices, a correlation factor $R$ was introduced. It is defined by $R=I C 1_{\text {air }} / I C 2_{\text {air }}$ where $I C 1_{\text {air }}$ and $I C 2_{\text {air }}$ are the readings of the two devices without the sample. In practice, the correlation factor $R$ was measured in the following way: Various aluminum foil layers were placed at the sample position and $I C 1$ and IC2 were recorded. $R^{-1}$ was extrapolated from the intercept on the plot of $I C 2 / I C 1$ versus the number of layers in a semilogarithmic scale. The air scattering pattern $I_{\text {air }}$ was also recorded for data reduction.

To give a clear description of the experimental procedures and data reduction, we show a schematic of the main scattering setup in Figure 1. Mechanical tests were carried out with a

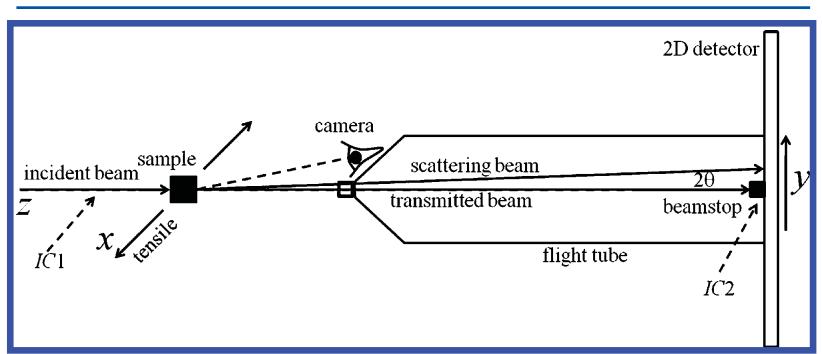

Figure 1. Schematic drawing of the experiment setup and the coordinates used in this work. The flight tube is under vacuum.

homemade tensile frame. This apparatus can stretch the sample in a symmetric fashion to guarantee the same position of the $\mathrm{X}$-ray spot on the sample at all extension ratios. To measure the local strain near the region hit by the X-ray beam, an alumina powder (analytical grade) was carefully deposited on the sample surface very close to the beam spot but not inside. A camera (PixeLINK B741EF, 1280 pixel $\times 1024$ pixel) was placed up the flight tube and tracked the displacement of the alumina particles (see Figure 1). Images were recorded every $0.5 \mathrm{~s}$ and synchronized with the stress-strain data. After the fracture of the sample, a background optical image was captured by the PixeLINK camera. An optical standard (NT62-212, Edmund Optics) with a $50 \mathrm{~mm} \times 50 \mathrm{~mm}$ dots pattern was placed in the clamps and the related image was also recorded for later data reduction. Samples were stretched along the $x$ axis (horizontal direction) with an initial strain rate of $0.25 / \mathrm{min}$ (about $12.5 \mathrm{~mm} / \mathrm{min}$ crosshead velocity). SAXS patterns were taken after each extension ratio increment $\Delta \lambda=0.1$ with an exposure time of $t(t=1 \mathrm{~s}$ for filled rubber samples in this work) and thus the relative change of elongation during each exposure was less than $4 \%$. No obvious radiation damage to the filled polymer was detected during the experiment.
3. Data Reduction. Measurement of Local Strain. The background was first subtracted from the optical images and then each image was corrected for the nonlinear effect (due to the lens) and for the perspective effect (due to the non parallelism between the focus plane and the object plane, see Figure 1). A free particle tracking package (developed by Daniel Blair and Eric Duresne, see http://physics.georgetown. $\mathrm{edu} / \mathrm{matlab} /$ ) for Matlab was used to obtain the local elongation in the $x$ direction in the vicinity of the beam spot.

SAXS Patterns. In order to obtain meaningful data when comparing materials, the measured raw intensity $I_{\text {measure }}$ (counts per second) needs to be corrected and normalized by several factors directly related both to the experimental setup and equipment being used and to the fact that during the experiment the sample thickness changed as it was strained.

The first correction to apply is the subtraction of the background which comes in two parts: the detector intensity pattern when the X-ray shutter is closed called $I_{\text {dark }}$ and the air scattering pattern from the air between the sample and the flight tube. The air scattering measured with no sample in the beam $\left(I_{\text {air }}\right)$ needs to be corrected by the attenuation due to the presence of the sample $($ RIC2/IC1) and by the ratio of incident beam intensity $\left(I C 1 / I C 1_{\text {air }}\right)$. The correction of air scattering is then:

$$
I_{\text {cor } 1}=I_{\text {measure }}-\frac{R I C 2}{I C 1} \frac{I C 1}{I C 1_{\text {air }}}\left(I_{\text {air }}-I_{\text {dark }}\right)-I_{\text {dark }}
$$

For the Pilatus 1 M 2D detector used no signal was detected when the X-ray shutter was closed, and hence $I_{\text {dark }}$ can be set to zero.

$I_{\text {corl }}$ needs to be corrected for changes in sample thickness $t_{\text {thick }}=\mu \operatorname{Ln}(I C 1 / R I C 2)$, and transmission $T=R I C 2 / I C 1$ and normalized by the incident beam intensity IC1.

$$
I_{\text {cor } 2}=\frac{I_{\text {cor } 1}}{F_{\text {Ins }} I C 1 t_{\text {thick }} T}=\frac{I_{\text {cor } 1}}{F_{I n s} \mu R I C 2 \ln (I C 1 / R I C 2)}
$$

with $F_{\text {Ins }}$ being an instrument factor and $\mu$ the X-ray absorption coefficient of the sample. Since we always compared the intensity at a given deformation to that of the same undeformed sample and used the same instrumental setup, $F_{\text {Ins }}$ and $\mu$ are neglected when computing the scattering pattern or scattering intensity. The scattering invariant $Q$ which is the integral of the corrected intensity $I_{\text {cor2 }}$ over all reciprocal space, is calculated with the assumption of cylindrical symmetry of the system along the loading direction ( $x$ axis) following the methodology developed for the analysis of crazes.

$$
\begin{aligned}
Q & =\frac{1}{2} \int_{-\infty}^{+\infty} \int_{0}^{\infty} I_{\text {cor } 2}\left(q_{x} q_{y}\right) q_{y} \mathrm{~d} q_{x} \mathrm{~d} q_{y} \\
& =\frac{1}{2} \frac{\int_{-\infty}^{+\infty} \int_{0}^{\infty} I_{\text {cor } 1}\left(q_{x} q_{y}\right) q_{y} \mathrm{~d} q_{x} \mathrm{~d} q_{y}}{R I C 2 \ln (I C 1 / R I C 2)}
\end{aligned}
$$

In this work, we always use the normalized scattering invariant $Q / Q_{0}$ (eq 4). The index 0 here represents the undeformed samples.

$$
\frac{Q}{Q_{0}}=\frac{\int_{-\infty}^{+\infty} \int_{0}^{\infty} I_{c o r 1}\left(q_{x} q_{y}\right) q_{y} \mathrm{~d} q_{x} \mathrm{~d} q_{y}}{\int_{-\infty}^{+\infty} \int_{0}^{\infty} I_{c o r 1.0}\left(q_{x} q_{y}\right) q_{y} \mathrm{~d} q_{x} d q_{y}} \frac{R I C 2_{0} \ln \left(I C 1_{0} / R I C 2_{0}\right)}{R I C 2 \ln (I C 1 / R I C 2)}
$$

Notice that the thickness $t_{\text {thick }}$ is measured by the X-ray absorption which will not be influenced by the presence of nanovoids. It will be different from the geometric thickness of 
the sample when nanocavitation is present. The intensity calculated by eq 2 is actually an intensity per unit thickness of solid material (the size of the beam spot is constant) and so are the scattering invariants $Q$ and $Q_{0}$.

To avoid the effect of the background noise in the high $q$ region, a radial cutoff $q$ value ( $q_{\text {cutoff }}$ ) is introduced above which the scattering intensity is neglected for the integration of both deformed and undeformed scattering patterns. The choice of $q_{\text {cutoff }}$ has almost no effect on the final result if a value larger than $0.8 \mathrm{~nm}^{-1}$ is used (see Supporting Information, SFigure 1). Thus, $q_{\text {cutoff }}$ is set to $0.8 \mathrm{~nm}^{-1}$ which is already well in the Porod's law regime where the intensity decreases as a power law with $q$.

In the raw scattering pattern, there are horizontal and vertical black bars which are the gaps between chip arrays on the Pilatus $1 \mathrm{M}$ detector (see Supporting Information, SFigure 2). We only used the right top quadrant and filled the black bars using scattering intensity in the other symmetrical positions for the calculation of $Q$. The scattering patterns shown in this work are also reconstructed from these right top quadrants after applying the corrections presented above.

4. The Three Phase Model. The primary goal of our scattering experiments is the detection of nanovoids during the tensile deformation of filled rubbers and for that purpose we used the scattering invariant. The scattering invariant $Q$ is directly related to the square of the X-ray scattering length density (XSLD) contrast between each phase, and is independent of the shape of the scatterers and of their spatial arrangement. ${ }^{44}$ The approach was originally developed to model a system containing multiple phases ${ }^{42,44-46}$ and here we use an analogous approach. Unlike the craze structure which is a typical two phase system (polymer and void), our vulcanizate contains not only polymer and filler, but also other additives (sulfur, 6PPD, CBS, stearic acid, and $\mathrm{ZnO}$ ). Here two important assumptions are made:

1 Additives are not taken into consideration because we assume that they are uniformly distributed in the rubber phase and while they may increase the X-ray scattering length density (XSLD) of this phase they do not give rise to a contribution to the invariant as a separate phase.

2 There are no preexisting nanocavities inside the undeformed samples, or more precisely, no preexisting nanocavities in the detectable length range via SAXS (5-250 $\mathrm{nm}$ in this work).

The samples were prepared under high pressure and well degassed, therefore the second assumption is reasonable and the scattering of the additives should not change during deformation. The scattering invariant for an ideal binary phase system without nanovoids is expressed by eq 5 according to small-angle X-ray scattering theory ${ }^{44}$

$$
\begin{aligned}
Q_{0} & =2 \pi^{2} V \phi_{S B R} \phi_{C B}\left(\rho_{S B R}-\rho_{C B}\right)^{2} \\
& =2 \pi^{2} V_{\text {solid }} \phi_{S B R} \phi_{C B}\left(\rho_{S B R}-\rho_{C B}\right)^{2}
\end{aligned}
$$

where $V$ is the volume of the scattering region and equals the volume of solid material $V_{\text {solid }}$ if no voids are present, $\phi_{\mathrm{SBR}}$ and $\phi_{\mathrm{CB}}$ denote the volume fractions of the polymer matrix and of the $\mathrm{CB}$ particles of the virgin samples and $\rho_{\mathrm{SBR}}$ and $\rho_{\mathrm{CB}}$ are the corresponding XSLD, respectively. If, upon mechanical loading, nanocavities appear, $Q$ is expected to increase due to the large XSLD contrast between nanovoids and their environment. For a three phase system similar to eq 5 , the invariant $Q$ can be determined via

$$
\begin{aligned}
Q= & 2 \pi^{2} V\left[\phi_{S B R, \lambda} \phi_{C B, \lambda}\left(\rho_{S B R}-\rho_{C B}\right)^{2}\right. \\
& \left.+\phi_{S B R, \lambda} \phi_{\text {void }} \rho_{S B R}{ }^{2}+\phi_{C B, \lambda} \phi_{\text {void }} \rho_{C B}{ }^{2}\right] \\
= & 2 \pi^{2} \frac{V_{\text {solid }}}{1-\phi_{\text {void }}}\left[\phi_{S B R \lambda} \phi_{C B \lambda}\left(\rho_{S B R}-\rho_{C B}\right)^{2}\right. \\
& \left.+\phi_{S B R, \lambda} \phi_{\text {void }} \rho_{S B R}{ }^{2}+\phi_{C B \lambda} \phi_{\text {void }} \rho_{C B}{ }^{2}\right]
\end{aligned}
$$

where, $\phi_{\mathrm{SBR} \lambda}, \phi_{\mathrm{CB} \lambda}$, and $\phi_{\text {void }}$ are the volume fraction of matrix, $\mathrm{CB}$ particles and nanovoids under external loading, respectively. ${ }^{42,45,46}$ Here, $V$ is no longer equal to the volume of solid material since nanovoids appear. The XSLD of the nanovoids is zero $\left(\rho_{\text {void }}=0\right)$ and the volume fractions $\phi_{S_{B R} \_} \lambda$ $\phi_{\mathrm{CB} \_} \lambda \phi_{\text {void }}, \phi_{\mathrm{SBR}}$ and $\phi_{\mathrm{CB}}$ are related by eqs $7 \mathrm{a}$ and $7 \mathrm{~b}$

$$
\begin{aligned}
& \phi_{S B R, \lambda}=\phi_{S B R}\left(1-\phi_{\text {void }}\right) \\
& \phi_{C B \lambda}=\phi_{C B}\left(1-\phi_{\text {void }}\right)
\end{aligned}
$$

By substituting eqs $7 \mathrm{a}$ and $7 \mathrm{~b}$ into eq 6 and dividing by eq 5 , an expression for the normalized invariant $Q / Q_{0}$ is obtained.

$$
\frac{Q}{Q_{0}}=1+\left[\frac{\phi_{S B R} \rho_{S B R}{ }^{2}+\phi_{C B} \rho_{C B}{ }^{2}}{\phi_{S B R} \phi_{C B}\left(\rho_{S B R}-\rho_{C B}\right)^{2}}-1\right] \phi_{\text {void }}
$$

In this work, $Q / Q_{0}$ was calculated from the measured scattering patterns and then the void volume fractions were calculated by using eq 8 .

5. Volume Variation Measurement by Digital Image Correlation. A similar dog bone shaped sample was marked with talc powder and stretched monotonically in the vertical direction with an Instron tensile machine with a cross head speed of $50 \mu \mathrm{m} / \mathrm{s}$. Two cameras were placed perpendicular to the front face and to the side face of the sample. Images of a central area $(7 \mathrm{~mm} \times 4 \mathrm{~mm}$ of front face, $7 \mathrm{~mm} \times 2.5 \mathrm{~mm}$ of side face) were recorded every $5 \mathrm{~s}$ and digital image correlation was then performed to calculate the macroscopic volume variation.

Digital image correlation ${ }^{47}$ was used to obtain the displacement field on the sample between the image taken at the beginning of the test and one taken during the test. Telecentric lenses were used to reduce the effect of out-of-plane displacement on our measured displacement fields. Those effects were measured and subtracted from the measured displacements.

The strain in the tensile direction plus either the front face transverse strain or the side face transverse strain are derived from those displacement fields. As expected, there were no noticeable differences between the measured strain in the tensile direction from the front face and the side face. The full deformation gradient tensor $\boldsymbol{F}$ is diagonal in our case. Its components are deduced from the derivation of the displacement fields measured with digital image correlation $(\nabla U)$.

$$
\begin{aligned}
& \nabla U=F-I \\
& F_{i, j}=\frac{\partial x_{i}}{\partial X_{j}}
\end{aligned}
$$

where $x_{i}$ are the coordinates in the deformed state and $X_{j}$ the coordinates in the initial state. The relative volume change is 
derived from the transformation gradient as

$$
\frac{\Delta V}{V}=\frac{\operatorname{det}(F)-1}{\operatorname{det}(F)}
$$

where $V$ is the volume after deformation and $\Delta V$ is the difference between the new and the initial volume $V_{0}$. The quantity $\Delta V / V$ has been selected to be comparable to the volume fraction of voids obtained by SAXS.

\section{RESULTS}

The results obtained from SAXS from CB-filled SBR under uniaxial tension are essentially of two kinds: the 2D scattering pattern as a function of applied elongation and the normalized scattering invariant calculated from eq 4 as a function of applied elongation. We chose to plot the data as a function of either true stress $\sigma_{\mathrm{T}}=F / S$ ( $F$ is the loading force, $S$ is the area of the cross section under deformation) or elongation $\lambda=l / l_{0}\left(l_{0}\right.$ is the initial gauge length of the sample and $l$ is the gauge length under deformation).

1. Un-Cross-Linked Sample. To verify the validity of the data reduction procedure and the cylindrical symmetry assumption, a filled but un-cross-linked sample 17CB_0NC was tested. This sample is essentially a highly filled viscoelastic fluid and contains the same cross-linking agents as the other samples but was carefully stored to avoid any chemical crosslinking. The chain entanglements are too weak to sustain high stresses. Thus, we do not expect nanocavitation to occur upon external loading and the scattering invariant is expected to remain constant as a function of $\lambda$. The stress $\sigma_{\mathrm{T}}$ and the normalized invariant $Q / Q_{0}$ curves are shown in Figure 2a. The true stress $\sigma_{\mathrm{T}}$ increases at small elongation but reaches a plateau

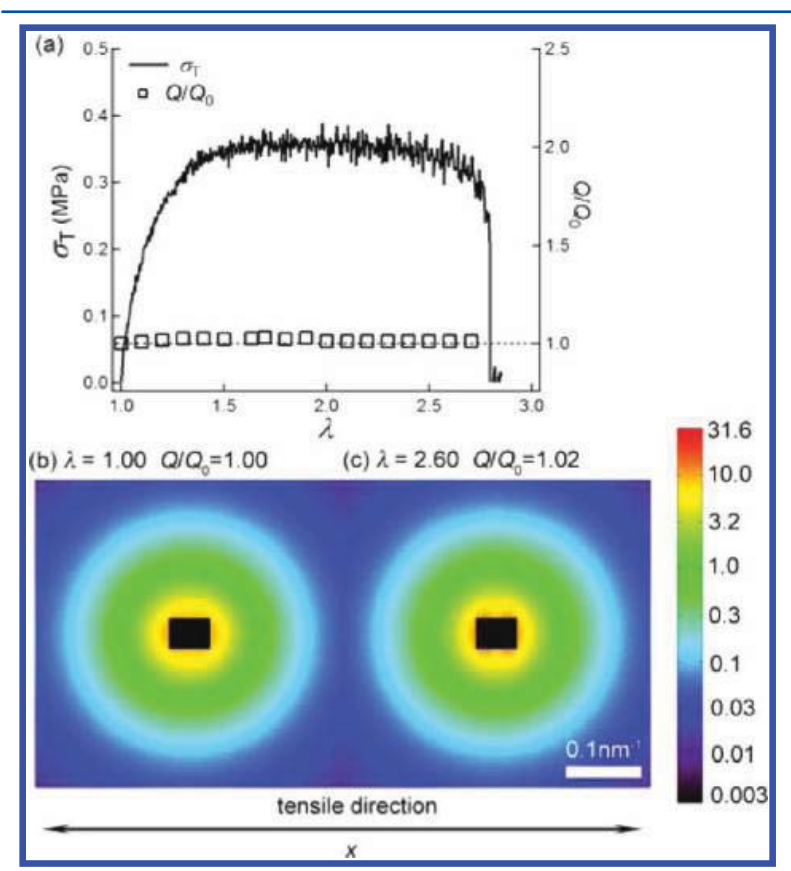

Figure 2. Results for an un-cross-linked sample 17CB_0NC. (a) True stress $\sigma_{\mathrm{T}}$ (line) and normalized scattering invariant $\mathrm{Q} / \mathrm{Q}_{0}$ (open symbols) as a function of $\lambda$. Experimental data were taken at ambient temperature $\left(25^{\circ} \mathrm{C}\right)$. The dashed line is just a guide to the eye. Selected scattering patterns at $(b)$ reference state $(\lambda=1)$ and $(c)$ close to the elongation at fracture where the log intensity (color scale) is that of intensity in arbitrary units. when $\lambda>1.5$. The stress-elongation curve is not very meaningful here since the sample exhibited necking at high extension. However the scattering data reveals some interesting information. In parts $\mathrm{a}$ and $\mathrm{b}$ of Figure 2, selected scattering patterns are shown. Before stretching, an isotropic pattern is observed reflecting the random dispersion of $\mathrm{CB}$ inside the rubber matrix. This pattern gradually changes with increasing strain to a butterfly shape in the low $q$ region with an intensity minimum perpendicular to the tensile axis. Very close to the fracture point (in the necked region), the butterfly pattern further evolves into a four-lobe pattern (data not shown here). Similar results have been reported for other filler-polymer systems ${ }^{29,30,34,48}$ (cross-linked or not), gels, ${ }^{49-51}$ and polymer melts. ${ }^{52}$ Although the detailed interpretation of such patterns is still unclear, it is generally accepted that it is the nonaffine relative displacement of the particles under deformation that accounts for the butterfly scattering pattern. CB aggregates are forced to come closer in the direction perpendicular to the tensile axis and cluster along narrow regions of higher $\mathrm{CB}$ particle density separated by highly deformed polymer in the direction parallel to the tensile axis. This clustering leads to a banded structure of $\mathrm{CB}$ aggregates lying at right angles to the tensile axis, and results in the observed butterfly scattering pattern. Yet, despite the change in the scattering pattern, $Q / Q_{0}$ remains constant at 1 which means that no new scattering phase appears during stretching as expected. Thus, our data reduction procedure and our assumption of cylindrical symmetry seem not to introduce any artifact into the analysis. To be complete one should mention that the uncertainty in $Q / Q_{0}$ for this un-cross-linked sample is \pm 0.02 , so fluctuation of any $Q / Q_{0}$ values below this range is regarded as indicating that no new scattering phase appears.

2. Nanocavitation in Cross-Linked Samples. We now show the results for the sample 24CB_8NC that has the highest volume fraction of filler. The key data are summarized in Figures 3, 4 and 5. In Figure 3a, $\sigma_{\mathrm{T}}$ and $Q / Q_{0}$ are plotted as a function of $\lambda$. The true stress increases monotonically with elongation and the sample breaks at a high extension ratio that is close to 5 . The invariant ratio $Q / Q_{0}$ remains equal to 1 over a rather large range of $\lambda$. Above a certain threshold value, $Q / Q_{0}$ begins to increase and reaches almost 2.5 times its original value before final fracture. This demonstrates the appearance of a third phase which exhibits a large XSLD contrast with its neighboring environment. Since SBR chains are unable to crystallize under external loading, small nanovoids represent the most probable third phase.

As shown in Figure $3 \mathrm{~b}, \sigma_{\text {onset }}$ was first determined from a $Q / Q_{0}$ vs $\sigma_{\mathrm{T}}$ plot (Figure $3 \mathrm{~b}$ ) and used to calculate $\lambda_{\text {onset }}$ from the related stress-elongation curves. For this sample, $\sigma_{\text {onset }}=$ $26.0 \mathrm{MPa}$ and $\lambda_{\text {onset }}=2.60$. The $Q / Q_{0}$ vs stress curve is quite reproducible for samples from the same batch (see Supporting Information, SFigure 3) and if an error exists in determining $\sigma_{\text {onset }}$ it is mainly due to the fact that we took exposures every $\Delta \lambda=0.1$ which will introduce an error about $\pm 2 \mathrm{MPa}$.

We also tried to detect the presence of nanocavities from the change in the scattering patterns. Typical scattering patterns are shown in Figure 4 and the corresponding scattering intensities along the $x$ and $y$ directions are also plotted in Figure 5. Without deformation, an isotropic pattern is observed, a signature of the scattering from the hierarchical structure of the $\mathrm{CB}$ filler aggregates in the rubber matrix (Figure $4 a$ ). ${ }^{53}$ The scattering intensity from the $\mathrm{CB}$ particles is composed of a Guinier's law region coming from $\mathrm{CB}$ aggregates (fusion of primary particles) 


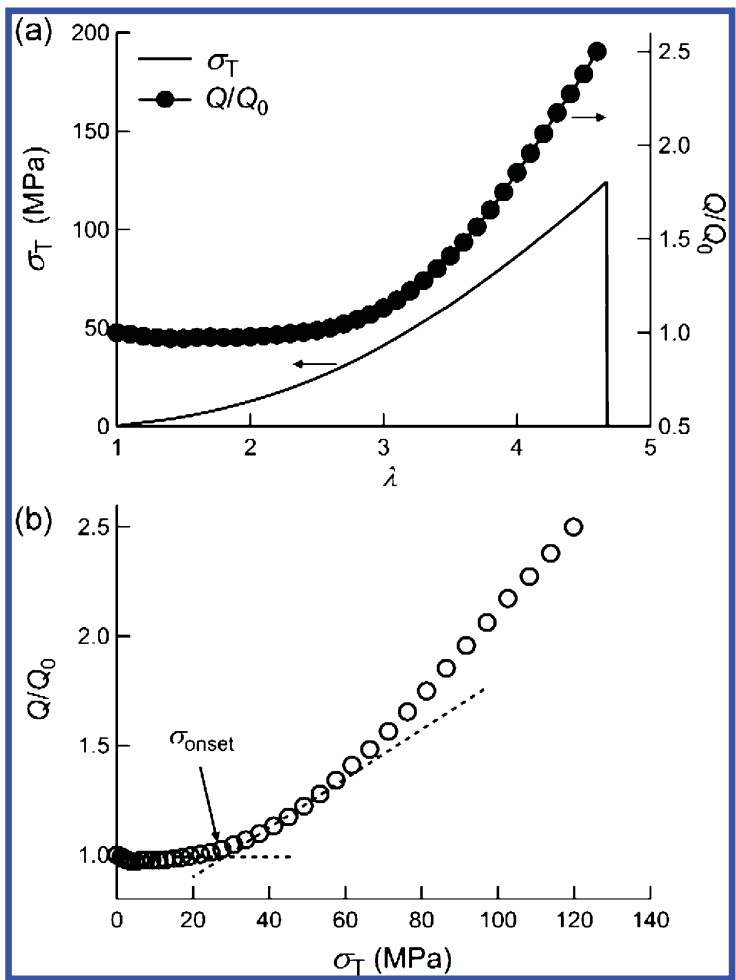

Figure 3. Scattering and tensile testing data of sample 24CB_8NC (a) $\sigma_{\mathrm{T}}$ (line) and $Q / Q_{0}$ (filled circle) are presented as a function of $\lambda$. (b) $Q / Q_{0}$ versus $\sigma_{\mathrm{T}}$. The void onset stress $\sigma_{\text {onset }}$ is determined from the intersection, indicated by the arrow, of two lines, one corresponding to $Q / Q_{0}=1$ and one tangent to the increasing $Q / Q_{0}$ curve.

and a Porod's law region that is due to the interface scattering between the primary particles and the matrix. Here the power law exponents are -3.67 and -3.65 in the $x$ and $y$ direction respectively, consistent with literature values. ${ }^{53}$

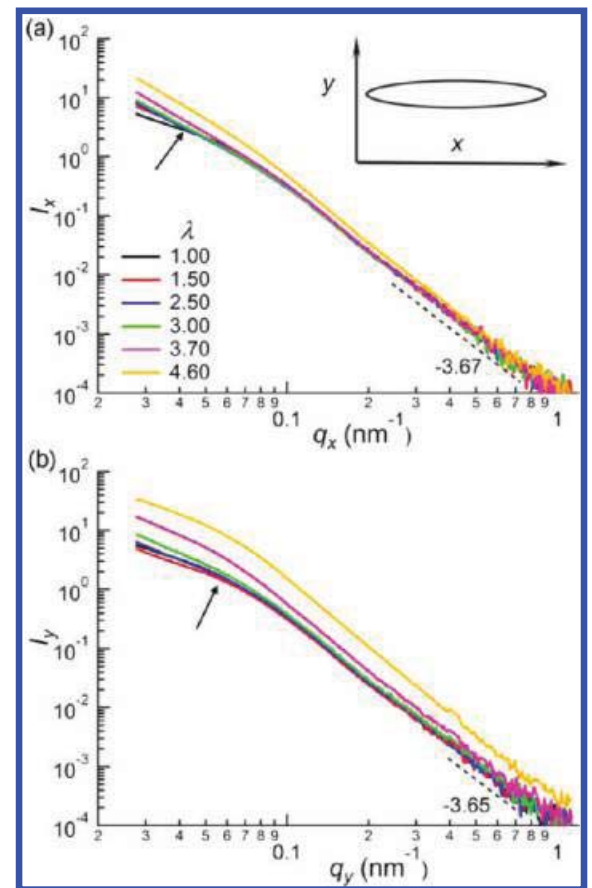

Figure 5. Scattering intensity (arbitrary units) as a function of $q$ along the $x(\mathrm{a})$ and $y(\mathrm{~b})$ directions at different values of $\lambda$ indicated in the legend for sample 24CB_8NC. The numbers close to the dashed lines are the power law exponents in the Porod's region. Arrows indicate the $q$ positions where scattering intensity starts to increase near $\sigma_{\text {onset }}$. The inset in part a is a schematic drawing of the elongation of nanovoids in the stretching direction.

At small $\lambda$ before nanocavitation occurs (Figure $4 b$ ), the pattern in the low $q$ region weakly splits along the tensile direction and tends to form a faint butterfly scattering pattern. The corresponding 1-D intensity in Figure 5, parts a and b (red lines), slightly decreases in the low $q$ region in the $y$ direction

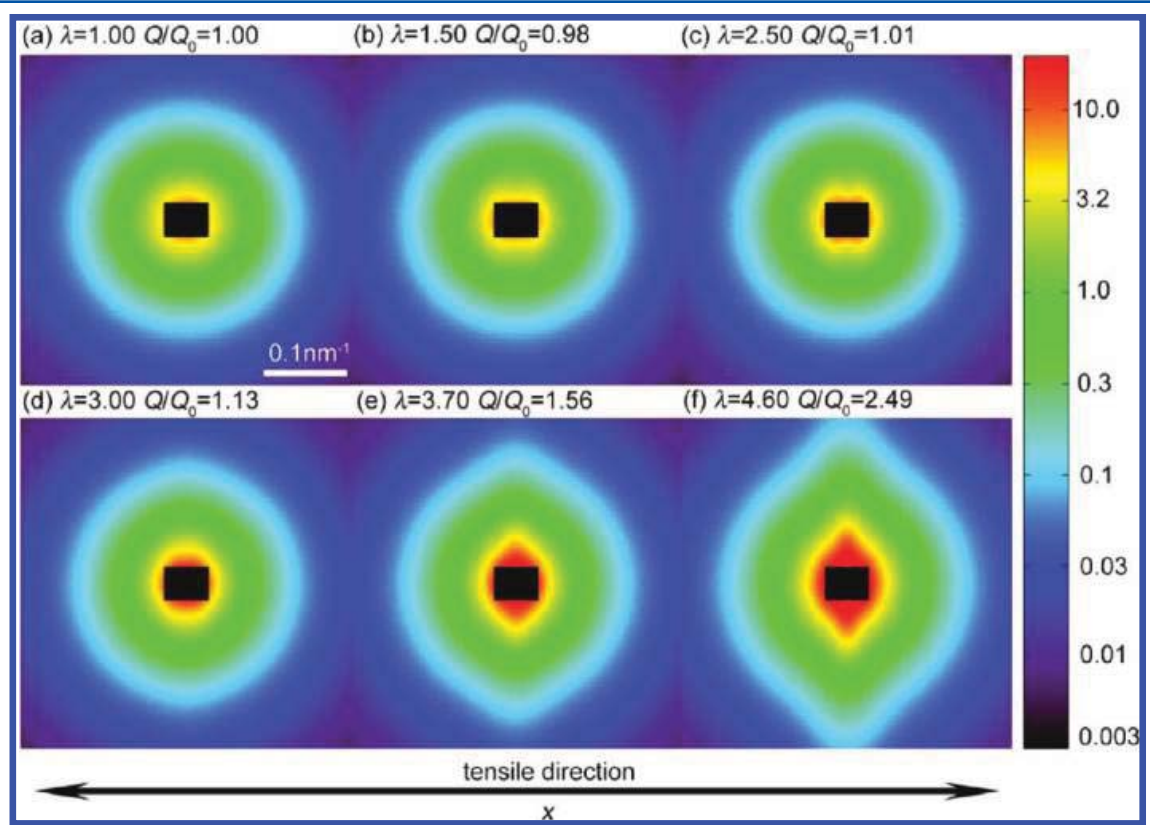

Figure 4. $(\mathrm{a}-\mathrm{f})$ Selected scattering patterns of sample $24 \mathrm{CB} \_8 \mathrm{NC}$ at different values of $\lambda$ as indicated in each frame title together with the corresponding $Q / Q_{0}$. The colors represent a logarithmic scale of scattered intensity (arbitrary units). 
but increases in the $x$ direction as was observed in the un-crosslinked specimen. The associated $Q / Q_{0}$ remains constant so this faint butterfly pattern is not related to the formation of nanovoids but rather to the nonaffine deformation of the filler aggregates.

At intermediate elongations before (Figure 4c) and after (Figure $4 \mathrm{~d}$ ) $\lambda_{\text {onset }}$ the valley in the $y$ direction in the low $q$ region is superimposed on the scattering pattern from nucleated nanocavities and gradually forms an elliptical pattern. However, this kind of change is not as sharp in other samples examined. Thus, we chose $Q / Q_{0}$ versus $\sigma_{\mathrm{T}}$ plots to determine the threshold stress and elongation rather than trying to directly estimate them from the SAXS patterns. In this range of $\lambda$, the intensity scattered by the nanovoids at initiation is more or less uniformly distributed along the $x$ and $y$ direction (blue and green lines in Figure 5) which apparently indicates that the initial shape of these nanovoids is nearly spherical. However, we think this is a false impression resulting from the superposition of the scattering from the particles and the weak scattering from the voids. As indicated by the arrows in Figure 5, the position of the crossover point is different in the $x$ direction $\left(\sim 0.4 \mathrm{~nm}^{-1}\right)$ and in the $y$ direction $\left(\sim 0.6 \mathrm{~nm}^{-1}\right)$ which suggests a more elongated structure in the tensile direction. Since the low $q$ region is associated with relatively larger length scales, it is expected that the sizes of these first nanovoids is rather large. A more detailed estimate of their size and shape will be presented in the Discussion.

At higher elongation, a sharp streak develops in the high $q$ region along the $y$ axis (Figure 4, parts $\mathrm{e}$ and $\mathrm{f}$ ) strongly suggesting the existence of highly elongated nanovoids with their major axis lying along the tensile direction (see the schematic drawing in Figure 5a). In the following we will refer to this pattern as a "flame pattern". The increase of the intensity is more pronounced in the $y$ direction even in the very high $q$ region though less significant in the tensile direction ( $x$ axis). This indicates the decrease of the average size of the nanovoids in both directions. Combined with the $Q / Q_{0}$ plot (Figure 3 ) we may deduce a broad size distribution of nanocavities (see Discussion for details). In addition, the surface fractal dimension (Porod's law region) of the $\mathrm{CB}$ aggregates in both directions at high $q(q>0.3)$ remains unchanged which is consistent with Schneider's observations in filled PDMS. ${ }^{31}$

The volume fraction of nanovoids $\phi_{\text {void }}$ was calculated from eq 8. The XSLD of the SBR matrix and of the CB filler particles are $\rho_{\mathrm{SBR}}=8.756 \times 10^{10} \mathrm{~cm}^{-2}$ and $\rho_{\mathrm{CB}}=15.26 \times 10^{10} \mathrm{~cm}^{-2}$, respectively. The curve (open symbols in Figure 6) is quite similar to the $Q / Q_{0}$ plot in Figure 3a. Above $\lambda_{\text {onset }} \phi_{\text {void }}$ grows up to $11 \%$ before fracture occurs. Such a large volume fraction of nanocavities should be easily detectable via a macroscopic volume variation measurement. This was carried out by deforming identical tensile samples to the same extension ratios while measuring the volume change with $\lambda$ of a representative volume in the center of the sample with a digital image correlation (DIC) method.

A representative result is illustrated in Figure 6a. To clearly compare between the two solutions, we did not show the data points before the onset of cavitation for either curve. The similarity between both measurements is striking. The main discrepancies are the later onset of cavitation $\left(\lambda_{\text {onset }}=2.8\right)$ and a higher amount of total volume variation from macroscopic measurement, e.g., at $\lambda=4.00, \Delta V / V=8 \%$ while $\phi_{\text {void }}$ from SAXS is $6 \%$. The difference between the two parameters can be explained by the relative advantages and limitations of the two

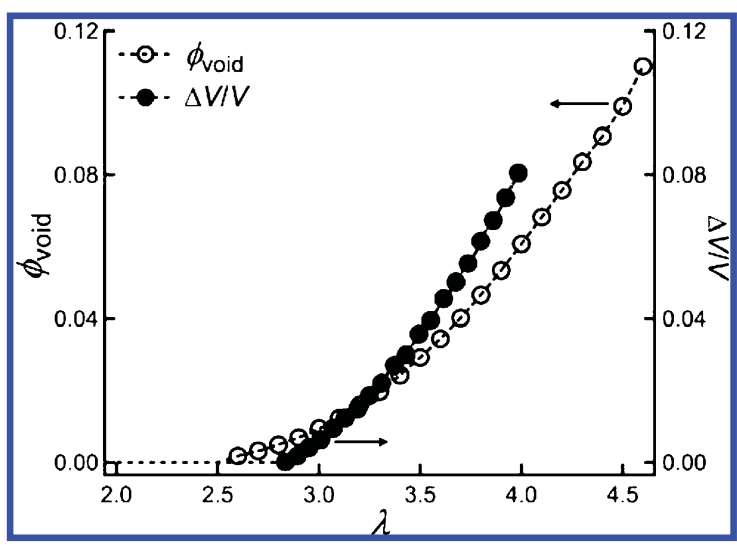

Figure 6. $\Delta V / V$ obtained from DIC measurement (right axis, filled symbols) and void volume fraction $\phi_{\text {void }}$ (left axis, open symbol) calculated from the three phase model are plotted as a function of elongation for comparison. The data points which are just fluctuations around zero before the onset of cavitation for both curves are not shown for clarity.

methods and by the uncertainty in our interpretation of the orientation effect at high strain.

Because of the large electron density contrast between the nanovoids and the other two phases, SAXS is more sensitive to the onset of nanocavitation but not as accurate at high deformation for the following reason: The scattering invariant is the sum of the contribution of smaller nanovoids entering into the observation window minus bigger ones exiting it; the latter scatter primarily at very low $q$ region and can be hidden under the beamstop. The sensitivity of the detection can be estimated by eq 8 which is linear in $\phi_{\text {void }}$. For example for sample $24 \mathrm{CB}$ 8NC $0.5 \%$ of voids results in $7 \%$ increase in $Q /$ $Q_{0}$ which is well above our detection threshold. On the contrary, well conducted macroscopic volume variation measurements can provide the global volume change more precisely at high deformation because all cavities, regardless of their size are accounted for, but is less accurate for the $\lambda_{\text {onset }}$ depending on the details of the method being used. That can explain the higher cavitation threshold value and lower amount of volume variation from the DIC measurement at the initiation of cavitation $(\lambda<3.2)$ as well as the crossing of the two curves in Figure 6. Even at higher elongations the difference between the two methods is small which quantitatively demonstrates the capability of our three phase model. In another filler-rubber systems studied by volume variation measurement alone, a value of global volume change was estimated to a similar range. 19

3. Effect of Filler Volume Fraction. All samples shown in Figure 7 have almost the same cross-linking density but different filler volume fractions. In Figure $7 \mathrm{a}, \sigma_{\mathrm{T}}$ is plotted as a function of $\lambda$ for various filler volume fractions. In agreement with established data ${ }^{1}$ the increase in filler volume fraction increases the modulus and decreases somewhat the maximum extensibility. $\phi_{\text {void }}$ is represented as a function of $\lambda$ in Figure $7 \mathrm{~b}$ for all four materials and $\lambda_{\text {onset }}$ decreases clearly with increasing $\phi_{\mathrm{CB}}$ as shown in Figure $7 \mathrm{~d}$. Interestingly, however, if $\phi_{\text {void }}$ is plotted as a function of $\sigma_{\mathrm{T}}$ (Figure 7c), a constant $\sigma_{\text {onset }}$ of approximately $25 \mathrm{MPa}$ is measured when $\phi_{\mathrm{CB}}>14 \%$ (Figure $7 \mathrm{~d}$ ). This value is much higher than the cavitation stress observed in bulk unfilled rubber stretched under confinement ${ }^{10,14}$ but a similar value has also been predicted to trigger cavitation 


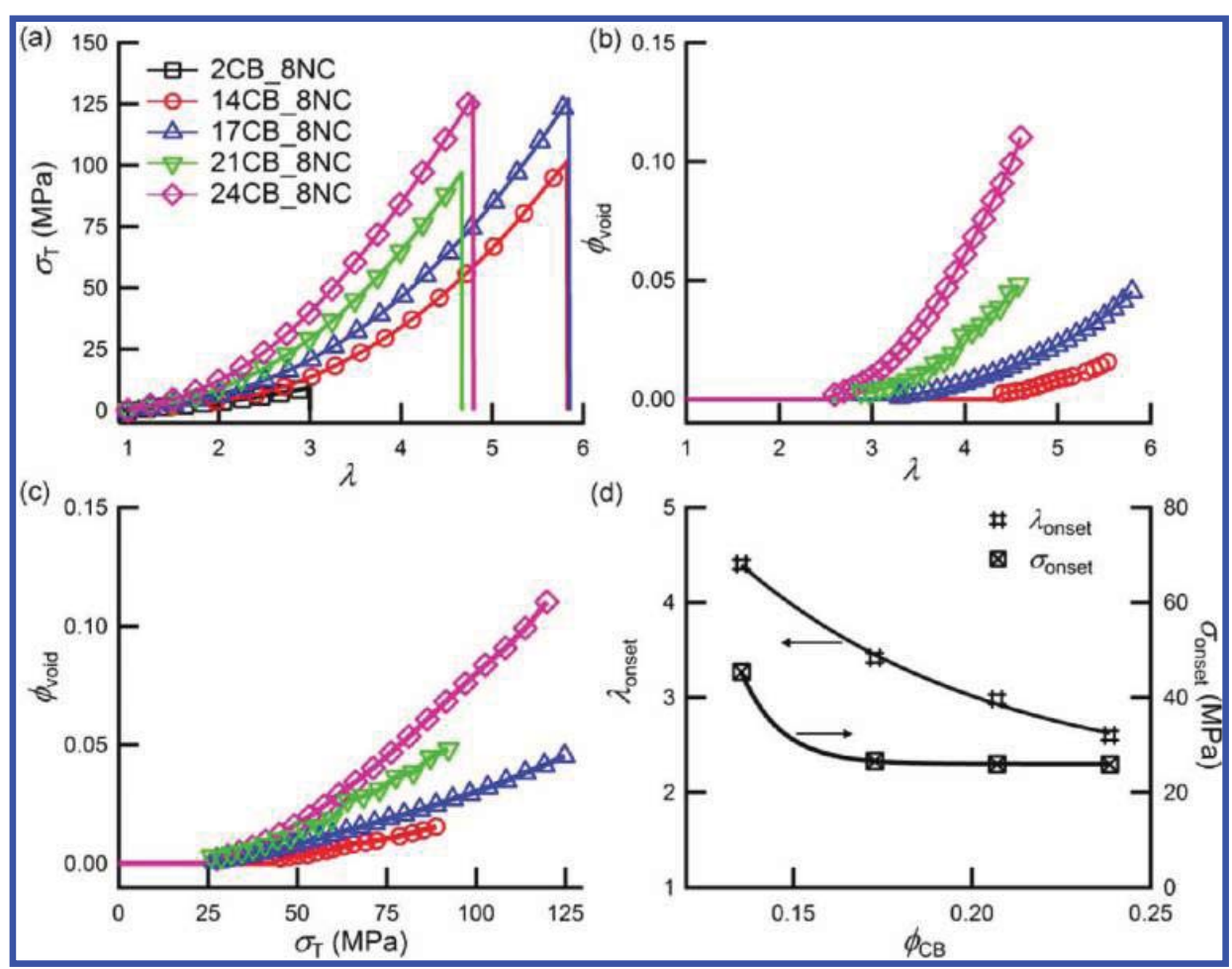

Figure 7. Effect of filler volume fraction on the nanocavitation of CB-SBR systems. (a) Stress-elongation curves. (b and c) Void volume fraction $\phi_{\text {void }}$ as a function of $\lambda$ and $\sigma_{\mathrm{T}}$ respectively. (d) $\lambda_{\text {onset }}$ and $\sigma_{\text {onset }}$ as a function of filler volume fraction $\phi_{\mathrm{CB}}$. Lines in part d are simply guides to the eyes. The data points that are just fluctuations around zero before the onset of cavitation for all curves in parts $\mathrm{b}$ and $\mathrm{c}$ are not shown for clarity.

of the rubber phase in toughened plastics. ${ }^{54,55}$ For 14CB_8NC, this $\sigma_{\text {onset }}$ shifts to a higher value of approximately $45 \mathrm{MP}$. After initiation, $\phi_{\text {void }}$ grows in a more linear fashion with $\sigma_{\mathrm{T}}$. The result obtained for the sample with a very low filler content (2CB_8NC) may be representative of unfilled elastomers. For this low $\phi_{\mathrm{CB}}$ sample, $Q / Q_{0}$ does not increase upon stretching and thus no significant cavitation at the nanometer length scale is detected before macroscopic fracture occurs. The fact that observable nanocavitation requires the presence of a certain amount of filler implies that the level of local confinement of the matrix induced by the filler aggregates plays a critical role.

Some additional information can be inferred from the pattern itself. In Figure 8, typical 2D scattering patterns and related $Q / Q_{0}$ are shown for different samples at different levels of elongation. In the lightly filled $2 \mathrm{CB} 8 \mathrm{NC}$, an elongated scattering pattern was observed just before fracture at $\lambda=3$. The scattering intensity increased along the $y$ axis but decreased along the $x$ axis, resulting in no variation in the scattering invariant $\left(Q / Q_{0}=0.97\right)$. This shows that the presence of an elliptical pattern per se does not indicate the presence of nanovoids and must be combined with the measurement of $Q / Q_{0}$.

For those samples where an increase in $Q / Q_{0}$ is observed, SAXS patterns similar to those in Figure 4 are observed for both 17CB_8NC and 21CB_8NC with a transition from isotropic patterns to weak butterfly patterns and then to an elliptical shape and finally to flame patterns. As for $14 \mathrm{CB} \_8 \mathrm{NC}$, the weak butterfly pattern is not detected and elliptical patterns are observed directly after the onset of nanocavitation.

4. Effect of Cross-Linking Density. All samples in Figure 9 have the same filler volume fraction but different cross-linking density. Like the increase in filler volume fraction, increasing the cross-linking density results in a higher modulus of the filled rubber as shown in Figure 9a. However this increase in crosslinking density has little effect on the initiation of nanocavitation. Only a slight variation in $\lambda_{\text {onset }}$ is seen in Figure $9 \mathrm{~b}$. If $\phi_{\text {void }}$ is plotted as a function of $\sigma_{\mathrm{T}}$ in Figure 9c, $\sigma_{\text {onset }}$ has a weak dependence on the cross-linking density. Interestingly, there is no distinct difference between the $17 \mathrm{CB} \_8 \mathrm{NC}$ and the $17 \mathrm{CB}$ 11NC samples in terms of nanocavitation (red and blue lines) but a notable difference in fracture elongation. An even higher cross-linking density (17CB_15NC) results in an earlier brittle rupture and hinders the nucleation of nanocavities. Probably the stress required to nucleate the nanocavities is close to or even higher than the stress to propagate a crack through the sample. Results from Figures 7 and 9 are insufficient to draw any correlations between the amount of voids just before fracture and the elongation at fracture. In some cases, the system may form many small nanovoids without coalescence thus allowing it to extend to high elongation before fracture. In other cases, the nanovoids may coalesce or develop into crack-like shapes even at low void volume fractions. In Figure $9 \mathrm{~d}, \lambda_{\text {onset }}$ and $\sigma_{\text {onset }}$ are summarized as a function of network cross-linking density $\nu_{\mathrm{c}}$ and compared with the prediction of the macroscopic cavitation theory based on an elastic instability ${ }^{10}$ which is indicated by the red dashed line in Figure 9d.

Concerning the scattering pattern, there are no significant differences between the two less cross-linked samples $17 \mathrm{CB} 4 \mathrm{NC}$ and $17 \mathrm{CB} 8 \mathrm{NC}$ (Figure 10 and Figure 8). The well-developed streak perpendicular to the tensile direction is observed in both cases, indicating the presence of highly elongated nanovoids parallel to the stretching axis. However, in the more cross-linked 17CB_11NC, a low eccentricity elliptical scattering pattern is present from the onset of 


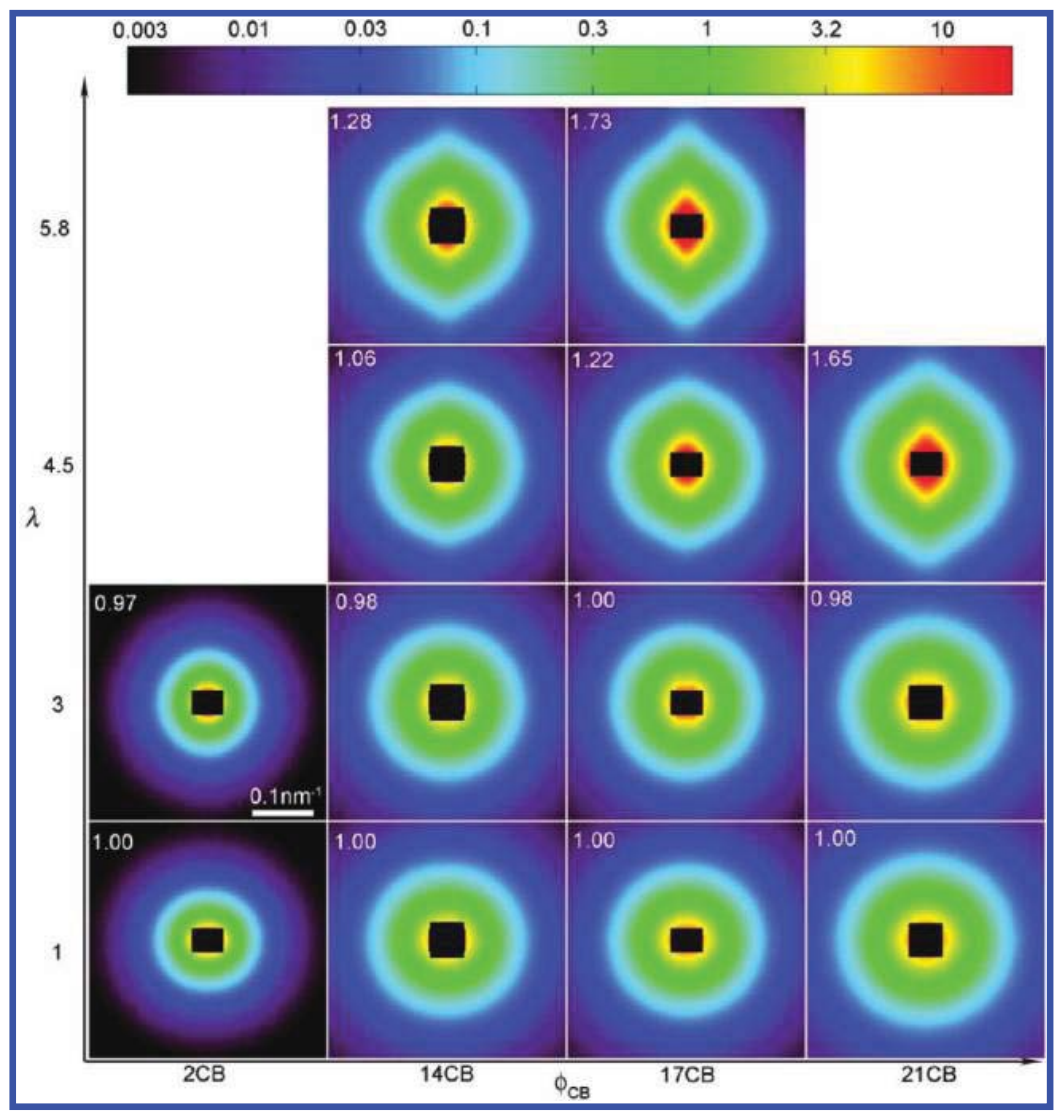

Figure 8. Effect of filler volume fraction on the scattering patterns. The vertical axis is elongation $\lambda$ and the horizontal axis is filler volume fraction $\phi_{\mathrm{CB}}$. The number in each image indicates the related normalized scattering invariant $Q / Q_{0}$. Color codes are the same as in Figure 4 and are shown on a log scale.
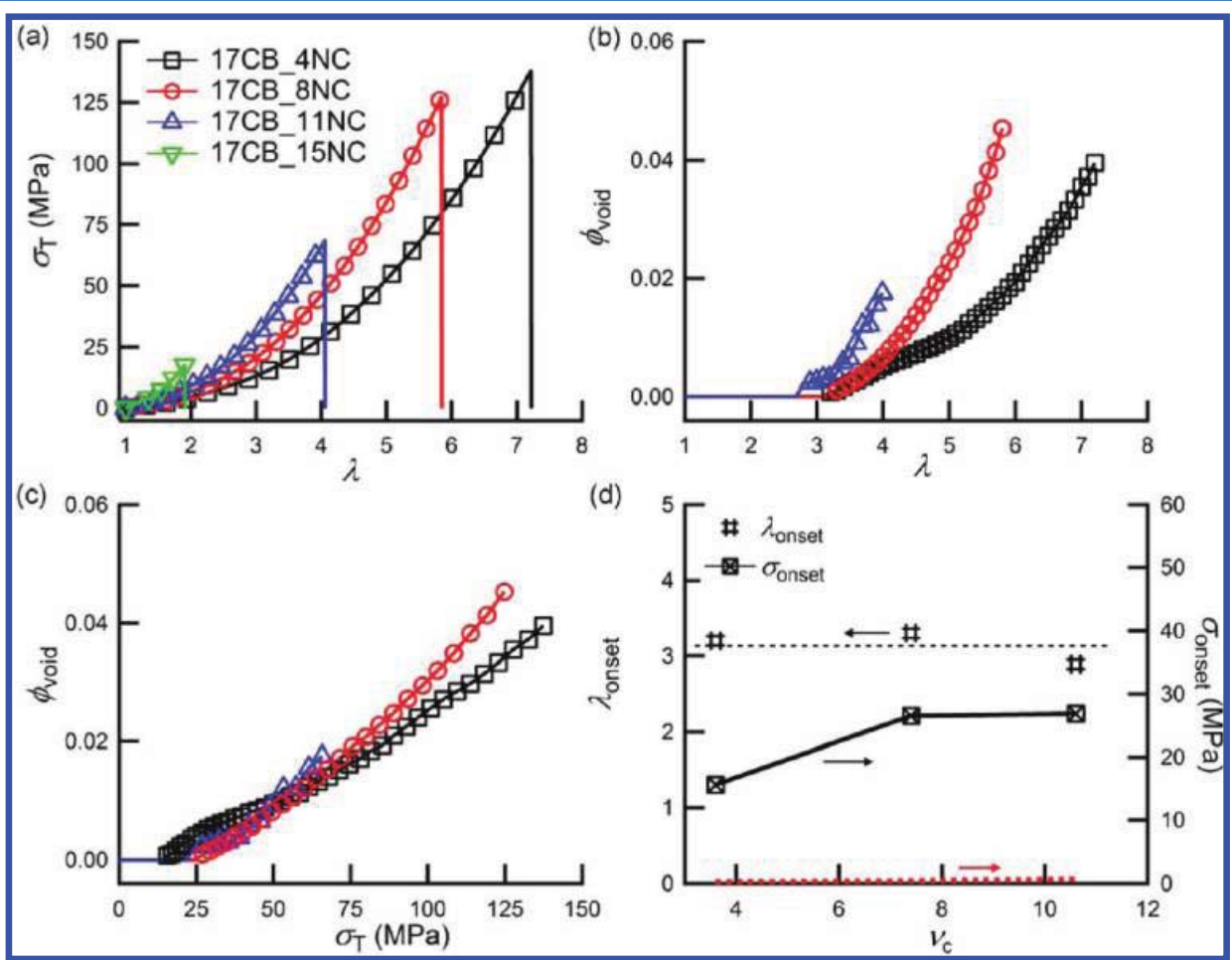

Figure 9. Effect of cross-linking density on the cavitation of CB-SBR systems. (a) Stress-elongation ratio curves. (b and c) Void volume fraction $\phi_{\text {void }}$ as a function of $\lambda$ and $\sigma_{\mathrm{T}}$ respectively. (d) $\lambda_{\text {onset }}$ and $\sigma_{\text {onset }}$ as a function of network cross-linking density $\nu_{\mathrm{c}}\left(\times 10^{-5} \mathrm{~mol} / \mathrm{cm}^{3}\right)$. The black dashed line in d is just a guide for the eyes while the red dashed line is the prediction of cavitation stress based on an elastic instability (eq 17 and see the Discussion for more details). The data points which are just fluctuations around zero before the onset of cavitation for all curves in parts $\mathrm{b}$ and $\mathrm{c}$ are not shown for clarity. 


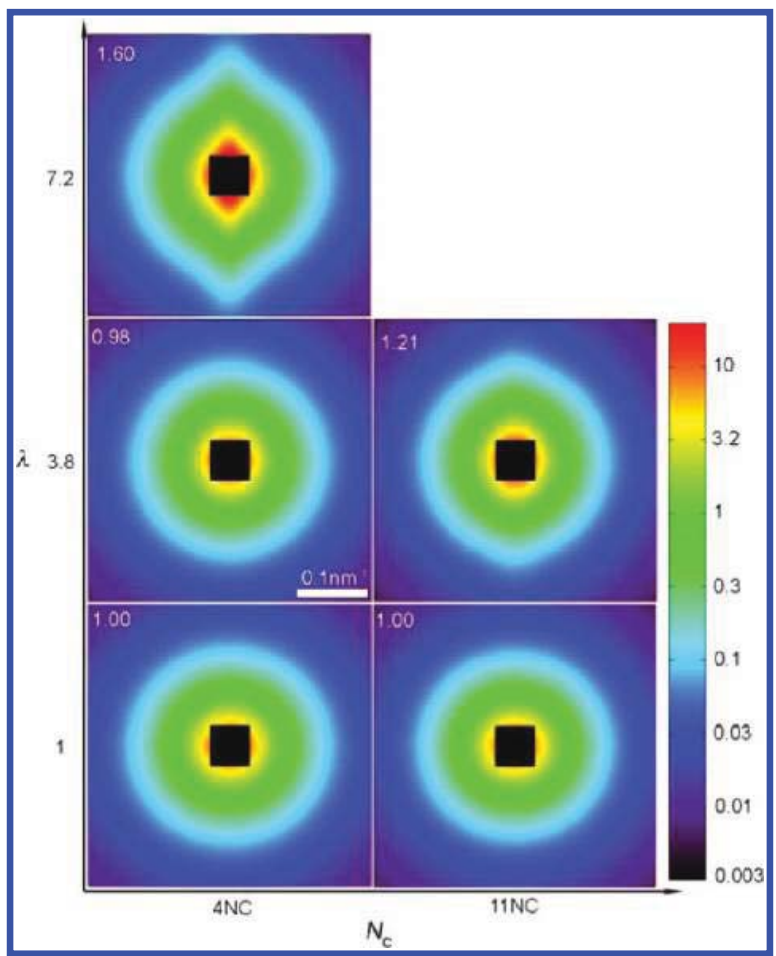

Figure 10. Effect of cross-linking on the scattering patterns. The vertical axis is the elongation $\lambda$ and the horizontal axis is the network cross-linking density $\nu_{\mathrm{c}}$. The number in each pattern indicates the related normalized scattering invariant $Q / Q_{0}$. The color bar is the same as that in Figure 4.

nanocavitation to the final rupture (Figure 10). The orientation of nanovoids is moderate in this case, probably hindered by the cross-linking of the matrix, and the whole sample is quite brittle. For the even more highly cross-linked $17 \mathrm{CB}$ 15NC, no nanocavitation is seen before fracture and the scattering patterns are very similar to those observed for the lightly filled 2CB_8NC.

\section{DISCUSSION}

1. The Three Phase Model and SAXS. Possible volume changes in filled rubbers have been studied for many years ${ }^{8,19,21,56}$ and have been reviewed by Le Cam. ${ }^{18}$ A positive volume variation is often ascribed to cavitation or decohesion at the filler-matrix interface or to fracture of the elastomer network while a negative volume change is related with crystallization under strain. While crystallization under strain is well documented and well understood, nanocavitation in uniaxial tension has not been conclusively demonstrated and quantified. The SAXS technique and in particular the analysis of the scattering invariant using the three phase model that we have developed, offers a way to quantitatively compare the SAXS volume change measurements with macroscopic volume change measurements. A few assumptions deserve to be discussed: One may doubt the hypothesis that there are no small preexisting voids inside the sample within the size range detectable via SAXS $(5-250 \mathrm{~nm}$ in the present work). All materials were prepared under pressure and well degassed and no evidence of preexisting nanovoids is seen from electron microscopy observations. Furthermore, if such voids were present we would expect these nanovoids to grow even at low elongation and increase $Q / Q_{0}$ which we did not observe. The main limitation of SAXS is the lack of sensitivity to large voids. Once nanovoids coalesce to form micrometer size voids at high strain, their scattering signal is hidden under the beam stop and no longer contributes significantly to the scattering invariant. So the macroscopic volume variation should always be larger than that is detected by SAXS. More consistency between the SAXS and macroscopic volume change measurements could be obtained if a much lower $q$ region was attainable in SAXS, e.g. by using a longer sample to detector distance. Unfortunately this distance was limited in these experiments to $4 \mathrm{~m}$ due to the hutch dimensions at beamline 7.3.3 at ALS.

2. Size of Nanovoids. To obtain an estimate of the size of these nanovoids, the scattering from nanovoids needs to be separated from the total scattering. Perfect deconvolution is very difficult here because the formation of $\mathrm{CB}$ aggregates also depends on the void shape and their dispersion state inside the sample, i.e., the two forms of scatterings (from $\mathrm{CB}$ and from nanovoids) are coupled. But we can make an estimate based on the following assumptions:

1 The onset of nanocavitation occurs in the low $q$ region which is associated with a length scale larger than the primary $\mathrm{CB}$ particles. Thus, only the $\mathrm{CB}$ near the boundaries of the voids modifies the scattering.

2 Except in the $y$-direction, the scattering changes only slightly in the high $q$ region under external stretching, even after the appearance of nanovoids (see Figure 4, 8, and 10). It is therefore acceptable to assume an isotropic scattering from $\mathrm{CB}$. Actually, $\mathrm{CB}$ filler clusters are believed to be relatively "stiff" at the smaller length scales, ${ }^{2}$ consistent with a relatively unaltered scattering pattern in the high $q$ region.

Under these two assumptions, the scattering from the voids $I_{\text {void }}$ can be obtained from the following equation:

$$
I_{\text {void }}=I_{\text {total }}-I_{C B}^{0}
$$

where $I_{\text {total }}$ is the total scattering and $I^{0}{ }_{\mathrm{CB}}$ is the scattering from $\mathrm{CB}$ just before nanocavitation. In Figure 11a, the scattering patterns of $I_{\text {void }}$ from sample 24CB-8NC are shown. Actually, the shape of the flame pattern is closer to that of a lozenge than to an ellipse. A similar lozenge shape was also observed in another sample using the same procedure. SANS "lozenge" scattering patterns are often seen in stretched polymer networks with dilute deuterated chains, ${ }^{57,58}$ but these are thought to result from the superposition of scattering from the stretched chains between cross-links and that from relaxed dangling chain ends. Here the lozenge shape of the scattering pattern clearly results from the shape of the voids themselves. Large lozenge or diamond shaped cavities are often observed in the ductile fracture of (strain hardened and thus oriented) polycarbonate $^{59,60}$ and polyvinyl chloride. ${ }^{61,62}$

In order to extract size information, a classical Porod analysis $^{44}$ based on approximating the actual lozenge-ofrevolution shape by an ellipsoid was applied here to $I_{\text {void }}$ to calculate the size of the voids.

$$
\frac{\left\langle R^{3}\right\rangle}{\left\langle R^{2}\right\rangle}=\frac{3 \lambda_{\text {void }}^{2}}{\pi\left(1-\phi_{\text {void }}\right)} \frac{Q_{\text {void }}}{K_{p y}}
$$

$R$ is the radius of a sphere with the same volume as the ellipsoid and $\lambda_{\text {void }}$ describes the deformation from a spherical shape. $Q_{\text {void }}$ is the scattering invariant from nanovoids and $K_{P y}$ is the Porod's constant along the $y$ axis. Other parameters are 


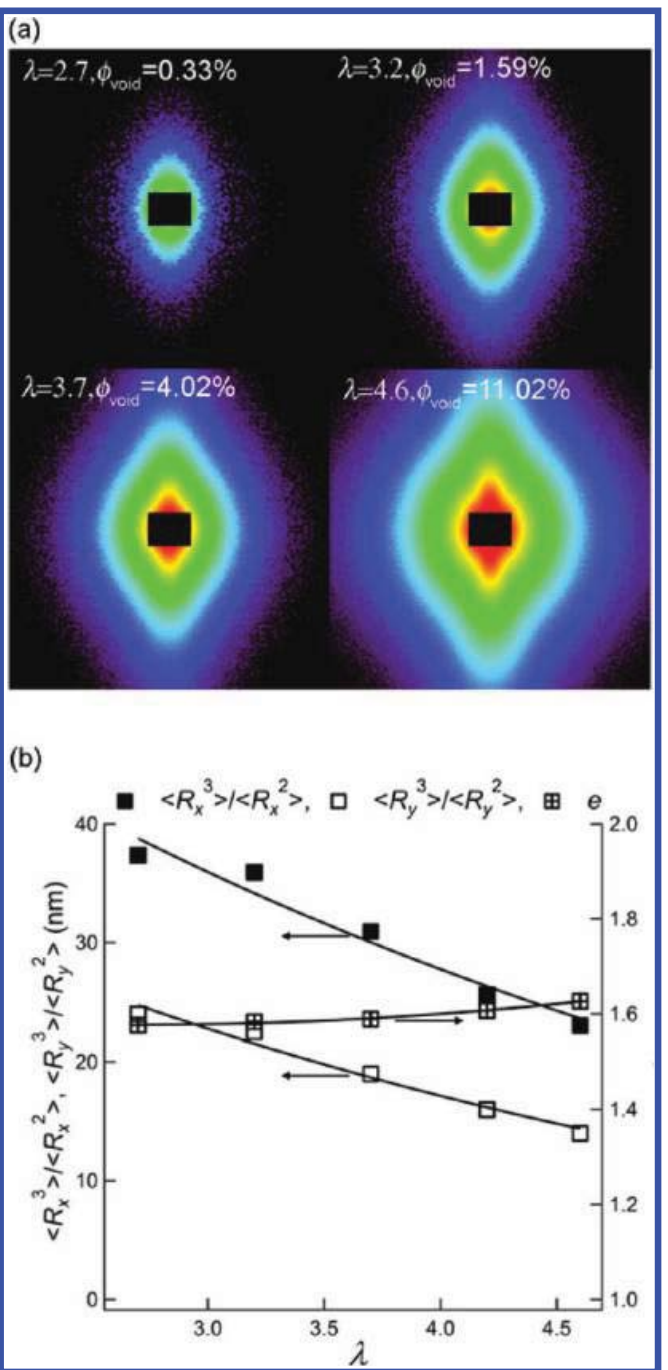

Figure 11. Estimate of the size of nanovoids for sample 24CB_8NC by eq 14 and 15 . (a) Selected $I_{\text {void }}$ (from eq 12) scattering patterns, from nanovoids at different elongations. The color scale is the same as that in Figure 4. (b) The average radius of voids in the $x$ and $y$ direction $\left(\left\langle R_{x}^{3}\right\rangle /\left\langle R_{x}{ }^{2}\right\rangle\right.$, filled squares) and $\left(\left\langle R_{y}^{3}\right\rangle /\left\langle R_{y}^{2}\right\rangle\right.$, open squares) as well as their ratio $e$ (eq 16, right axis) are plotted as a function of the elongation ratio $\lambda$. Black lines are just guides to the eyes.

then expressed by:

$$
\begin{aligned}
& \frac{\left\langle R_{x}{ }^{3}\right\rangle}{\left\langle R_{x}{ }^{2}\right\rangle}=\frac{\left\langle R^{3}\right\rangle}{\left\langle R^{2}\right\rangle}=\lambda_{\text {void }} \\
& \frac{\left\langle R_{y}{ }^{3}\right\rangle}{\left\langle R_{y}{ }^{2}\right\rangle}=\frac{\left\langle R^{3}\right\rangle}{\left\langle R^{2}\right\rangle} \frac{1}{\sqrt{\lambda_{\text {void }}}}
\end{aligned}
$$

The aspect ratio $e$ is defined as:

$$
\frac{\left\langle R_{x}{ }^{3}\right\rangle}{\left\langle R_{x}{ }^{2}\right\rangle} \frac{\left\langle R_{y}{ }^{2}\right\rangle}{\left\langle R_{y}{ }^{3}\right\rangle}=\lambda_{\text {void }}{ }^{3 / 2}
$$

The detailed derivation of these expressions is in the Appendix and the result of the analysis for sample 24CB_8NC is plotted in Figure 11b.
The average size is obtained from the average volume to surface ratio $\left(\left\langle R_{x}{ }^{3}\right\rangle /\left\langle R_{x}{ }^{2}\right\rangle\right.$ and $\left.\left\langle R_{y}{ }^{3}\right\rangle /\left\langle R_{y}{ }^{2}\right\rangle\right)$. As the elongation increases from $\lambda=2.5$ to $\lambda=4.5$, this average size decreases in both directions, from 37 to $25 \mathrm{~nm}$ in the $x$ direction and from 24 to $15 \mathrm{~nm}$ in the $y$ direction. However, the average aspect ratio $e$ (defined by eq 16) of these nanovoids is larger than 1 (implying an elliptical shape) even at the initiation stage and remains nearly constant implying that the average shape of the nanovoids hardly changes as $\lambda$ increases. If the increase of $\phi_{\text {void }}$ at high elongation was mainly due to the growth of existing voids, the average size in the tensile direction should have increased with elongation, which is not observed. Therefore, Figure $11 \mathrm{~b}$ clearly confirms the appearance of additional smaller voids at larger $\lambda$, consistent with the observations in Figure 5.

3. Cavitation Criterion in CB-SBR On the basis of an Elastic Instability. Macroscopic cavitation in confined bulk unfilled elastomers is thought to originate from the unbound expansion or fracture of preexisting defects under a strong local hydrostatic tensile stress. ${ }^{54}$ Green and Zerna ${ }^{63}$ showed that the hydrostatic stress $P_{\mathrm{C}}$ needed to inflate a large spherical vacuole surrounded by neo-Hookean material is related to the Young's modulus $E$ of the elastomer network by:

$$
P_{\mathrm{C}}=5 / 6 E_{\text {network }}
$$

This result, valid for a constant applied hydrostatic true stress and for an infinitely large sample was surprisingly confirmed experimentally in Gent's early papers. ${ }^{10,64}$ We use here the word surprisingly because the simple mechanical treatment of Green and Zerna did not include the surface tension of the rubber $\gamma$, which will introduce a closing pressure $p=2 \gamma / r$ and increase the critical expansion stress of cavities of initial ratio smaller than $\gamma / E$, and only considered reversible expansion (no fracture).

Gent and Tompkins took into account the surface tension ${ }^{64}$ and could explain some discrepancies between experiments and predictions. However, for our materials an estimate of the initial defect size necessary to account for a surface tension controlled expansion stress of $25 \mathrm{MPa}$ is around $3 \mathrm{~nm}$ in radius $(\gamma$ is usually taken as $0.03 \mathrm{~J} / \mathrm{m}^{2}$ for SBR). Notice in Figure 11a, the average radius of the expanded cavities near the onset of cavitation is $\sim 30 \mathrm{~nm}$. Such an expansion would require a stretch around the nucleating defects of 10 , obviously exceeding the limiting stretch of the chains. Therefore, one needs to consider irreversible fracture as a mechanism of cavitation.

Several models have been proposed to account for the expansion by irreversible fracture of a preexisting void, ${ }^{13,54,65-68}$ which is physically reasonable for cross-linked rubbers. Gent suggested ${ }^{13}$ that the cavitation stress should be a nonlinear function of the ratio $G_{c} / r_{0} E_{\text {network }}$, where $G_{c}$ is the fracture toughness in uniaxial tension of the rubber, $E_{\text {network }}$ is the modulus of the rubber and $r_{0}$ is the radius of the initial defect. $^{13,14,17}$ In unfilled rubbers this model predicts the correct trends but the maximum measured values of the cavitation stress $\left(\sim 3 E_{\text {network }}\right)$ suggest the existence of large defects of the order of micrometers which are not observed experimentally prior to cavitation. In our system, we do not observe macroscopic cracks and the measured $\sigma_{\text {onset }}$ of nanocavitation is at least ten times the Young's modulus of the rubbery matrix $(\sim 2 \mathrm{MPa})$ and nearly three times the best results obtained with unfilled systems relative to their modulus. We believe that the reason for this discrepancy between the threshold observed for unfilled systems (where the confined region is much larger than 
the defect size) and our system (where both sizes are comparable) cannot be explained by the previously described models, which assume an infinite size for the sample, and in the following we attempt to explain the high value of nanocavitation stress and its dependence on filler volume fraction and cross-linking density (Figures 7 and 9) by a finite size effect. Although many approximations are made, we feel that the discussion following can be the basis for improvement by future experiments and simulations.

\section{NANOCAVITATION IN CB-SBR BASED ON AN ENERGY BALANCE}

Although the macroscopic stress we applied is uniaxial and uniform, the CB aggregates can disturb the stress field locally and create regions of radius $R_{c o n}$ (presumably between filler particles) where the rubber matrix is strongly confined. As a result, a local hydrostatic expansion stress can be present in these confined regions between fillers. This local hydrostatic stress will vary spatially in magnitude and presumably depend on the spatial organization of the hard filler particles. It is difficult to quantitatively relate the macroscopic tensile stress to the distribution of local hydrostatic stresses but if the degree of local confinement remains moderate, they should have the same order of magnitude.

All models discussed in the previous section are valid for large confined volumes where the growing cavity size is much smaller than the confined volume. Yet in filled elastomer systems, the confined volumes (between particle aggregates) are of the same size as the growing nanocavities and are dispersed in a material which is predominantly in uniaxial tension. This has two consequences: First as soon as a nanocavity nucleates, its growth releases local confinement and prevents further growth by removing the driving force. This delays coalescence of the cavities and macroscopic fracture of the material. Second the growth criterion must include the size of the confined domain and not only the local stress. We propose here a model inspired from cavitation in rubber-filled thermoplastics where nanodomains under hydrostatic tension coexist with zones in uniaxial tension. In this model adapted to very small cavities, both surface tension and fracture toughness play a role.

In rubber toughened plastics, the effect of rubber particle size on particle cavitation has long been observed and discussed. Some researchers have reported that the cavitation process in rubber particles cannot occur when the diameter of the rubber particle decreases below $200 \mathrm{~nm}$ even if the local stress state would allow the initiation of voids. This indicates that both the local stress and the total strain energy available to drive cavitation play key roles. For example, to form a void with a radius of $r_{0}$, the local hydrostatic stress should exceed an inner pressure $P_{\text {surf }}$ related to the surface tension of the polymer by:

$$
P_{\text {local }}>P_{\text {surf }} \quad \text { with } \quad P_{\text {surf }}=2 \gamma / r_{0} \quad \text { and } \quad r_{0}>2 \gamma / P_{\text {local }}
$$

The energy barrier $U_{\text {barrier }}$ used to create such a void is ${ }^{69,70}$

$$
U_{\text {barrier }}=4 \pi \gamma r_{0}^{2}>16 \pi \gamma^{3} / P_{\text {local }}^{2}
$$

Here, the $U_{\text {barrier }}$ is underestimated because the energy to fracture the cross-linked chains along the inner surface and to stretch the surrounding polymer chains should also be considered.

Several models based on an energy balance were proposed to account for cavitation of rubber particles in rubber toughened plastics or to predict cavity growth in soft adhesive layers. In those models where finite size is important, the key parameter controlling cavitation is the local volumetric strain energy rather than the hydrostatic stress (volumetric strain energy density). But none of them considered the effect of the volume fraction of fillers which will alter the dependence of the local strain energy on the external loading. To qualitatively explain nanocavitation in our CB-SBR system, we can use an energy-based model developed by Fond et al. ${ }^{70}$ In their model, $\sigma_{\text {onset }}$ is given by:

$$
\sigma_{\text {onset }}=\frac{C}{f} R_{c o n}{ }^{-3 / 4} K^{1 / 4} \gamma^{1 / 2}\left(\gamma+G_{c}\right)^{1 / 4}
$$

where $f$ is a geometrical factor relating the local hydrostatic stress to the external loading, $R_{\text {con }}$ is the radius of the confined rubber domains (see Figure 12a), $K$ is the bulk modulus of the

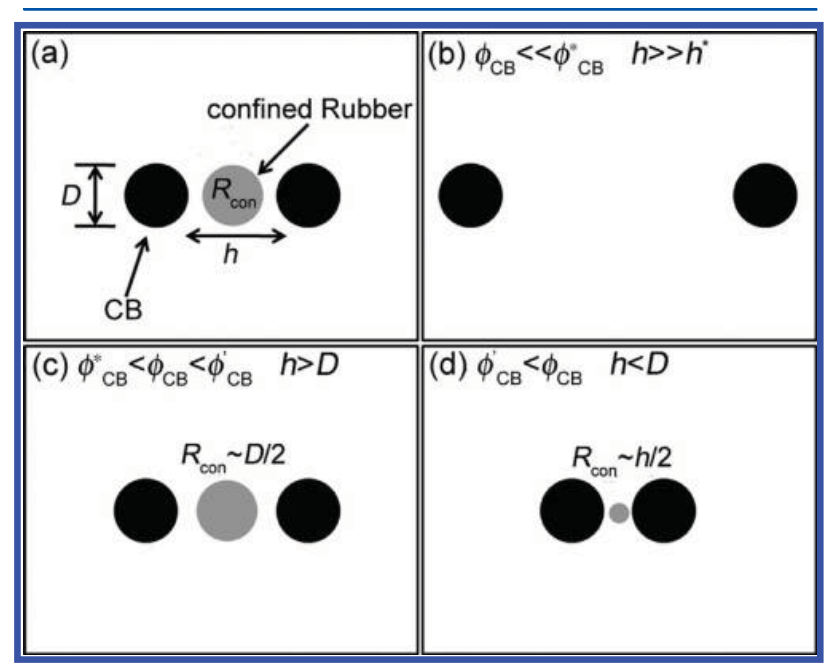

Figure 12. Schematic drawing of the filler volume fraction effect on cavitation. (a) Definition of parameters. (b) Drawing in the dilute region where there is little mechanical interaction between fillers. (c) In the moderate filler volume fraction, the volume of the confined rubber domains is unaffected. (d) In the highly filled region, the volume of the confined rubber domains depends on the interparticle distance $h$.

rubber, $\gamma$ and $G_{c}$ are the surface tension and fracture toughness of the rubber, respectively. $C$ is just a numerical factor and is not important for this qualitative description. An obvious conclusion from eq 20 is that $\sigma_{\text {onset }}$ does not depend on Young's modulus but on the bulk modulus and surface tension $\gamma$ which are nearly independent of the degree of cross-linking. Only $\mathrm{G}_{\mathrm{c}}$ is indirectly related to the cross-linking density.

It is quite reasonable since we have mentioned that the inner pressure due to surface tension which depends on van der Waals forces rather than cross-linking density is quite important to initiate nanovoids. Equation 20 also shows that $\sigma_{\text {onset }}$ depends strongly on $R_{\text {con }}$ which implies that for very small confined rubber domains no cavitation should occur at the initial stage.

To interpret the effect of the volume fraction of filler, both the change of $f$ and that of $R_{\text {con }}$ with $\phi_{\text {Св }}$ must be described. Here we just consider the simplest situation where two filler particles (or filler aggregates) with diameter $D$ are separated by a distance $h$ (see Figure 12a) and the mechanical interaction 
due to other fillers is not considered. Under this condition, $f$ is a function of $D$ and $h$. The increase of $\phi_{\text {Св }}$ always decreases the average interparticle distance $h$ and increases the degree of confinement (i.e., larger $f$ ). Unlike in rubber toughened plastics, $R_{\text {con }}$ in our systems also depends on the local geometry. Three regimes can be defined roughly according to the value of $\phi_{\mathrm{CB}}$. $\mathrm{A}$ schematic drawing is illustrated in Figure $12 \mathrm{~b}-\mathrm{d}$.

(1) $\phi_{\mathrm{CB}} \ll \phi^{*}{ }_{\mathrm{CB}}$ and interparticle distance $h \gg h^{*}\left(\phi^{*}{ }_{\mathrm{CB}}\right.$ and $h^{*}$ relate to the point where the particle stress fields start interacting). The filler particles are so "dilute" that no mechanical interaction is present. There is no geometric confinement except very close to the particles, ${ }^{7}$ but because the hard particle is very small as well as the associated volume of confined rubber domain, so a very large stress is required for cavitation (see Figure 12b). In this regime and in macroscopically uniaxial tension, nanocavitation, if it exists at all, is probably immediately followed by crack propagation.

(2) $\phi^{*}{ }_{\mathrm{CB}}<\phi_{\mathrm{CB}}<\phi_{\mathrm{CB}}^{\prime}$ and interparticle distance $h>D\left(\phi_{\mathrm{CB}}^{\prime}\right.$ is the point where the $h=D)$. The volume of the confined domains is almost of the order of the size of the filler particles $\left(R_{\text {con }} \sim D / 2\right)$ (Figure $12 \mathrm{c}$ ) and does not change much with an increase of $\phi_{\mathrm{CB}}$. The increase of $f$ (or of the local hydrostatic stress) dominates the nanocavitation behavior. Consequently, the local volumetric strain energy increases with $\phi_{\mathrm{CB}}$ and the apparent nanocavitation stress $\sigma_{\text {onset }}$ decreases with filler volume fraction.

(3) $\phi_{\mathrm{CB}}^{\prime}<\phi_{\mathrm{CB}}$ and interparticle distance $h<D$. Now the volume of the confined rubber regions is no longer a constant value but related to the interparticle distance $h$ $\left(R_{\text {con }} \sim h / 2\right)$ and has the opposite effect to the increase in $f$ (Figure 12d). Thus, the increase of filler content in this regime does not obviously increase the local volumetric strain energy available for nanocavitation although it does increase the local volumetric strain energy density. An almost constant $\sigma_{\text {onset }}$ with $\phi_{\mathrm{CB}}$ is expected according to eq 20 .

This simple model qualitatively explains some of our observations, but the real nanocavitation process in filled rubbers at such small scales is probably more complicated and more efforts are still required to propose a quantitative model that can predict $\sigma_{\text {onset }} \phi_{\text {void }}$ and shape information on the nanovoids with material parameters. Nevertheless our results and analysis show that these nanocavities exist and lay the groundwork to develop such a model.

Nanocavitation itself does not absorb a lot of energy but does reduce the local confinement. Under uniaxial loading conditions, nanocavitation and the orientation of nanovoids along the tensile direction modify the local stress field from locally multiaxial to nearly uniaxial everywhere which should lead to a more effective relaxation of high stresses. The loss of local triaxiality also greatly reduces the local strain energy release rate ${ }^{71}$ and prevents the growth of crack-like voids. It also probably promotes the reorganization of the filler particles along the direction of the maximum shear stress. A similar concept is also discussed in rubber toughened plastics where shear bands are often observed after rubber cavitation. ${ }^{72}$ In our CB-filled materials, both nanocavitation and particle rearrangement by shear are present. Local reorganization of particles can occur before and after nanocavitation depending on the fillerfiller interaction, filler-polymer interaction and organization of the aggregates. The cross-linking density of the rubber network could have an important effect on the above-mentioned scenario. For too highly cross-linked networks, the orientation of nanovoids as well as the rearrangement of filler particles could become quite difficult since stretching the highly oriented chains requires a much higher stress and involves a high risk of chain breakage.

Although the current work only focuses on uniaxial extension, it also provides clues for crack propagation. The most important role of nanovoids is to reduce the resistance of the local polymer matrix to volumetric expansion due to the hydrostatic stress field which often occurs at the crack tip. This could lead to the formation of a highly stretched zone near the crack tip over a larger volume than predicted by incompressibility.

\section{CONCLUSION}

We performed real-time SAXS experiments on a series of carbon black filled SBR elastomers stretched in uniaxial tension. For each value of stress and strain we recorded a $2 \mathrm{D}$ SAXS scattering pattern from which we extracted the scattering invariant $Q$ as a function of $\sigma_{\mathrm{T}}$ or $\lambda$. A sharp increase in $Q$ above its reference value $Q_{0}$ for the undeformed sample, was observed above a given value of $\lambda$ for all materials investigated except for the fully un-cross-linked and for the cross-linked but nearly unfilled elastomer. The $Q / Q_{0}$ increased above a threshold in true stress $\sigma_{\text {onset }}$ of the order of $25 \mathrm{MPa}$, which did not depend much on filler content or on the cross-link density of the elastomers. However, the subsequent increase in $Q / Q_{0}$ with $\sigma_{\mathrm{T}}$ beyond the threshold depended markedly on filler content and on cross-link density.

We attributed the increase in $Q / Q_{0}$ to the appearance of nanovoids and using the assumption of cylindrical symmetry and a three phase model, we computed the volume fraction of voids $\phi_{\text {void }}$ as a function of $\sigma_{\mathrm{T}}$ or $\lambda$. This volume fraction increased with $\lambda$ beyond $\lambda_{\text {onset }}$ and reached up to $15 \%$ at high extension ratios for the most highly filled system. The increase in volume expected from the appearance of voids and the value of $\sigma_{\text {onset }}$ was confirmed by macroscopic measurements of volume variation performed by digital image correlation demonstrating for the first time unambiguously the nucleation of nanovoids (size $<50 \mathrm{~nm}$ ) in CB-filled elastomers in uniaxial extension.

The 2D scattering patterns obtained as a function of $\lambda$ were further analyzed by assuming that the contribution of the $\mathrm{CB}$ particles and of the voids could be separated. The contribution of the voids to the scattering pattern at $\lambda>\lambda_{\text {onset }}$ displayed a lozenge shape which did not further change with $\lambda$. The average shape and size of the voids as a function of $\lambda$ could be determined from the surface to volume ratio of the scattering objects and revealed that as $\lambda$ increased the average size of the cavities decreased from 30 to $20 \mathrm{~nm}$ while their average shape remained constant. This clear result strongly suggests that new smaller cavities with similar shapes appear as $\lambda$ increases. The underlying mechanism(s) for the nanocavitation is still open to discussion but we think that the cavities appear in confined regions between filler particles and an energy based nanocavitation criterion inspired by the model of Fond et al. for the cavitation of rubber nanoparticles in rubber-filled thermoplastics is consistent with our data. Two main functions of those nanocavities are to alter the local multiaxial loading to a more uniaxial loading and to promote the rearrangement of the nearby filler aggregates by shear. 


\section{APPENDIX}

The deconvolution of the scattering intensity of nanovoids from the measured patterns is described below. The scattering from nanovoids $I_{\text {void }}$ is calculated from eq 9. The classical Porod's analysis is then applied to $I_{\text {void }}$. If we assume that the nanovoids are ellipsoids, according to Wen Li Wu's work ${ }^{73}$ for a single ellipsoid whose major axis is aligned with the $x$ direction, the Porod's constants take the following form. In the $x$ direction:

$$
K_{p x}=\lim _{q_{x} \rightarrow \infty} q_{x}^{4} I_{\text {void }}\left(q_{x}\right) \cong A \rho^{2}\left(8 \pi^{2} R^{2}\right) \lambda_{\text {void }}{ }^{-4}
$$

and in the $y$ direction,

$$
K_{p y}=\lim _{q_{y} \rightarrow \infty} q_{y}{ }^{4} I_{\text {void }}\left(q_{y}\right) \cong A \rho^{2}\left(8 \pi^{2} R^{2}\right) \lambda_{\text {void }}{ }^{2}
$$

where $A$ is the scattering constant, $\rho$ is the XSLD contrast between the ellipsoid and its environment, $R$ is the radius of a sphere with the same volume as the ellipsoid and $\lambda_{\text {void }}$ describes the deformation from a spherical shape. The ratios $K_{P y}$ and $K_{P x}$ directly give the value of $\lambda_{\text {void }}$. For a set of nanoellipsoids, eqs $\mathrm{A} 1$ and $\mathrm{A} 2$ are still applicable since the interparticle interference on the scattering intensity in the high $q$ region is negligible, ${ }^{44}$ but we must sum the scattering from $n_{\text {void }}$ where:

$$
n_{\text {void }}=\frac{3}{4 \pi} V \phi_{\text {void }} /\left\langle R^{3}\right\rangle
$$

Similar to eq 3 and 5, the scattering invariant $Q_{\text {void }}$ can be obtained and is related to the $\phi_{\text {void_Bi }}$ by:

$$
Q_{\text {void }}=2 \pi^{2} A \rho^{2} \phi_{\text {void }}\left(1-\phi_{\text {void }}\right) V
$$

If eq $\mathrm{A} 4$ is divided by the product of eqs $\mathrm{A} 2$ and $\mathrm{A} 3$, one gets:

$$
\frac{Q_{\text {void }}}{K_{\text {py }}}=\frac{1}{3} \pi\left(1-\phi_{\text {void }}\right) \frac{\left\langle R^{3}\right\rangle}{\left\langle\lambda_{\text {void }}{ }^{2} R^{2}\right\rangle}
$$

If the set of nanoellipsoids have roughly the same extent of deformation, $\left\langle R^{3}\right\rangle /\left\langle R^{2}\right\rangle$ is expressed by:

$$
\frac{\left\langle R^{3}\right\rangle}{\left\langle R^{2}\right\rangle}=\frac{3 \lambda_{\text {void }}^{2}}{\pi\left(1-\phi_{\text {void }}\right)} \frac{Q_{\text {void }}}{K_{p y}}
$$

other parameters are then expressed by

$$
\begin{aligned}
& \frac{\left\langle R_{x}{ }^{3}\right\rangle}{\left\langle R_{x}{ }^{2}\right\rangle}=\frac{\left\langle R^{3}\right\rangle}{\left\langle R^{2}\right\rangle}=\lambda_{\text {void }} \\
& \frac{\left\langle R_{y}{ }^{3}\right\rangle}{\left\langle R_{y}{ }^{2}\right\rangle}=\frac{\left\langle R^{3}\right\rangle}{\left\langle R^{2}\right\rangle} \frac{1}{\sqrt{\lambda_{\text {void }}}} \\
& \frac{\left\langle R_{x}{ }^{3}\right\rangle}{\left\langle R_{x}{ }^{2}\right\rangle} \frac{\left\langle R_{y}{ }^{2}\right\rangle}{\left\langle R_{y}^{3}\right\rangle}=\lambda_{\text {void }}{ }^{3 / 2}
\end{aligned}
$$

Finally, eqs A7-1-A7-3 are used as eq $14-16$ in the main text to plot Figure 11.

\section{ASSOCIATED CONTENT}

\section{S Supporting Information}

Influence of $q_{\text {cutoff }}$ (radial cutoff) on the calculation of the normalized scattering invariant $Q / Q_{0}$ of sample $24 \mathrm{CB} \_8 \mathrm{NC}$
(SFigure 1), the raw scattering pattern on the Pilatus $1 \mathrm{M}$ detector with horizontal and vertical stripes of sample $24 \mathrm{CB} \_8 \mathrm{NC}$ at $\lambda=4.6$ (SFigure 2), and the repeatability of the measurement of the onset of cavitation (SFigure 3 ). This material is available free of charge via the Internet at http:// pubs.acs.org.

\section{AUTHOR INFORMATION}

\section{Corresponding Author}

*E-mail: (C.C.) Costantino.Creton@espci.fr; (E.J.K.) edkramer@mrl.ucsb.edu.

\section{ACKNOWLEDGMENTS}

This work was supported by the French ANR: project AMUFISE, (MATETPRO 08-320101). A.K.S. and E.J.K. were supported by the Institute for Multiscale Materials Studies at UCSB, a collaboration with Los Alamos National Laboratory. We also appreciate the help of Eric Schaible at beamline 7.3.3 at ALS. The Advanced Light Source is supported by the Director, Office of Science, Office of Basic Energy Sciences, of U.S. Department of Energy under Contract No. DE-AC0205CH11231. We finally specially thank Stephane Roux for his critical reading of the manuscript and for helpful discussions and we acknowledge useful discussions with Daniel Berghezan, Yannick Merckel, Julie Diani, and Mathias Brieu.

\section{REFERENCES}

(1) Heinrich, G.; Kluppel, M.; Vilgis, T. A. Curr. Opin. Solid State Mater. Sci. 2002, 6 (3), 195-203.

(2) Kluppel, M. Adv. Polym. Sci. 2003, 164, 1-86.

(3) Kraus, G. Adv. Polym. Sci. 1971, 8, 155-237.

(4) Kohls, D. J.; Beaucage, G. Curr Opin Solid State Mater. Sci. 2002, 6 (3), 183-194.

(5) Gent, A. N.; Park, B. J. Mater. Sci. 1984, 19 (6), 1947-1956.

(6) Cho, K.; Gent, A. N. J. Mater. Sci. 1988, 23 (1), 141-144.

(7) Cho, K.; Gent, A. N.; Lam, P. S. J. Mater. Sci. 1987, 22 (8), 2899-2905.

(8) Oberth, A. E.; Bruenner, R. S. Trans Soc Rheol 1965, 9 (2), 165185.

(9) Oberth, A. E. Rubber Chem. Technol. 1967, 40 (5), 1337-1363.

(10) Gent, A. N.; Lindley, P. B. Proc. R. Soc. London A, Math. Phys. Sci. 1959, 249 (1257), 195-205.

(11) Farris, R. J. J. Appl. Polym. Sci. 1964, 8 (1), 25-35.

(12) Gee, G.; Stern, J.; Treloar, L. R. G. Trans. Faraday Soc. 1950, 46, 1101-1106.

(13) Gent, A. N.; Wang, C. J. Mater. Sci. 1991, 26 (12), 3392-3395.

(14) Cristiano, A.; Marcellan, A.; Long, R.; Hui, C. Y.; Stolk, J.; Creton, C. J. Polym. Sci. Polym. Phys. 2010, 48 (13), 1409-1422.

(15) Gent, A. N.; Tompkins, D. A. J. Polym. Sci. 2, Polym. Phys. 1969, 7 (9), 1483-1487.

(16) Horgan, C. O.; Polignone, D. A. Appl. Mech. Rev. 1995, 48 (8), 471-485.

(17) Lin, Y. Y.; Hui, C. Y. Int. J. Fract. 2004, 126 (3), 205-221.

(18) Le Cam, J. B. Rubber Chem. Technol. 2010, 83 (3), 247-269.

(19) Le Cam, J. B.; Toussaint, E. Mech. Mater. 2009, 41 (7), 898901.

(20) Starkova, O.; Aniskevich, A. Polym. Test. 2010, 29 (3), 310-318. (21) Ramier, J.; Chazeau, L.; Gauthier, C.; Stelandre, L.; Guy, L.; Peuvrel-Disdier, E. J. Mater. Sci. 2007, 42 (19), 8130-8138.

(22) Mzabi, S. Caracterisation et analyse des mecanismes de fracture en fatigue des elastomeres charges. Ph.D. Thesis, Universite Pierre et Marie Curie, Paris: 2010.

(23) Le Cam, J. B.; Huneau, B.; Verron, E.; Gornet, L. Macromolecules 2004, 37 (13), 5011-5017.

(24) He, C. B.; Donald, A. M.; Butler, M. F. Macromolecules 1998, 31 (1), 158-164. 
(25) Magalhaes, A. M. L.; Borggreve, R. J. M. Macromolecules 1995, 28 (17), 5841-5851.

(26) Humbert, S.; Lame, O.; Chenal, J. M.; Rochas, C.; Vigier, G. Macromolecules 2010, 43 (17), 7212-7221.

(27) Maier, G. A.; Wallner, G.; Lang, R. W.; Fratzl, P. Macromolecules 2005, 38 (14), 6099-6105.

(28) Pawlak, A.; Galeski, A. Macromolecules 2005, 38 (23), 96889697.

(29) Ehrburger-Dolle, F.; Bley, F.; Geissler, E.; Livet, F.; Morfin, I.; Rochas, C. Macromol. Symp. 2003, 200, 157-167.

(30) Ehrburger-Dolle, F.; Hindermann-Bischoff, M.; Livet, F.; Bley, F.; Rochas, C.; Geissler, E. Langmuir 2001, 17 (2), 329-334.

(31) Schneider, G. J. J. Chem. Phys. 2009, 130, 23.

(32) Schneider, G. J.; Goritz, D. J. Chem. Phys. 2010, 133, 2.

(33) Schneider, G. J.; Goritz, D. J. Appl. Crystallogr. 2010, 43, 12-16.

(34) Shinohara, Y.; Kishimoto, H.; Inoue, K.; Suzuki, Y.; Takeuchi, A.; Uesugi, K.; Yagi, N.; Muraoka, K.; Mizoguchi, T.; Amemiya, Y. J. Appl. Crystallogr. 2007, 40, S397-S401.

(35) Hagita, K.; Arai, T.; Kishimoto, H.; Umesaki, N.; Suno, H.; Shinohara, Y.; Amemiya, Y. Rheol. Acta 2008, 47 (5-6), 537-541.

(36) Hagita, K.; Arai, T.; Kishimoto, H.; Umesaki, N.; Shinohara, Y.; Amemiya, Y. J Phys, Condens. Matter 2007, 19, 33.

(37) Debye, P.; Anderson, H. R.; Brumberger, H. J. Appl. Phys. 1957, 28 (6), 679-683.

(38) Paredes, E.; Fischer, E. W. Makromol. Chem. 1979, 180 (11), 2707-2722.

(39) Paredes, E.; Fischer, E. W. J. Polym. Sci., Polym. Phys. 1982, 20 (5), 929-930.

(40) Brown, H. R.; Kramer, E. J. J. Macromol. Sci. Phys. 1981, B19 (3), 487-522.

(41) Mills, P. J.; Kramer, E. J.; Brown, H. R. J. Mater. Sci. 1985, 20 (12), 4413-4420.

(42) Brown, H. R.; Kramer, E. J.; Bubeck, R. A. J. Polym. Sci, Polym. Phys. 1987, 25 (8), 1765-1778.

(43) Flory, P. J. Principles of Polymer Chemistry; Cornell University Press: New York, 1953.

(44) Glatter, O.; Kratky, O. Small Angle X-ray Scattering; Academic Press: London, 1982; pp 19-51.

(45) Wu, W. Polymer 1982, 23 (13), 1907-1912.

(46) Cheung, Y. W.; Stein, R. S.; Lin, J. S.; Wignall, G. D. Macromolecules 1994, 27 (9), 2520-2528.

(47) Besnard, G.; Hild, F.; Roux, S. Exp. Mech. 2006, 46 (6), 789803.

(48) Le Diagon, Y.; Mallarino, S.; Fretigny, C. Eur. Phys. J. E 2007, 22 (1), 77-83.

(49) Mendes, E.; Lindner, P.; Buzier, M.; Boue, F.; Bastide, J. Phys. Rev. Lett. 1991, 66 (12), 1595-1598.

(50) Falcao, A. N.; Pedersen, J. S.; Mortensen, K. J. Mol. Struct. 1996, $383(1-3), 69-74$

(51) Mendes, E.; Oeser, R.; Hayes, C.; Boue, F.; Bastide, J. Macromolecules 1996, 29 (17), 5574-5584.

(52) Hayes, C.; Bokobza, L.; Boue, F.; Mendes, E.; Monnerie, L. Macromolecules 1996, 29 (14), 5036-5041.

(53) Koga, T.; Hashimoto, T.; Takenaka, M.; Aizawa, K.; Amino, N.; Nakamura, M.; Yamaguchi, D.; Koizumi, S. Macromolecules 2008, 41 (2), 453-464.

(54) Fond, C. J. Polym. Sci., Polym. Phys. 2001, 39 (17), 2081-2096.

(55) Gehant, S.; Fond, C.; Schirrer, R. Int. J. Fract. 2003, 122 (3-4), $161-175$.

(56) Le Cam, J. B.; Toussaint, E. Macromolecules 2008, 41 (20), 7579-7583.

(57) Read, D. J.; McLeish, T. C. B. Phys. Rev. Lett. 1997, 79 (1), $87-90$.

(58) Straube, E.; Urban, V.; Pyckhouthintzen, W.; Richter, D.; Glinka, C. J. Phys. Rev. Lett. 1995, 74 (22), 4464-4467.

(59) Donald, A. M.; Kramer, E. J. J. Mater. Sci. 1981, 16 (11), 29672976.

(60) Cornes, P. L.; Smith, K.; Haward, R. N. J. Polym. Sci., Polym. Phys. 1977, 15 (6), 955-967.
(61) Walker, N.; Haward, R. N.; Hay, J. N. J. Mater. Sci. 1979, 14 (5), 1085-1094.

(62) Walker, N.; Haward, R. N.; Hay, J. N. J. Mater. Sci. 1981, 16 (3), $817-824$.

(63) Green, A. E.; Zerna, W. Q. J. Mech. Appl. Math. 1950, 3 (1), 9-22.

(64) Gent, A. N.; Tompkins, D. A. J. Appl. Phys. 1969, 40 (6), 25202525.

(65) Dollhofer, J.; Chiche, A.; Muralidharan, V.; Creton, C.; Hui, C. Y. Int. J. Solids. Struct. 2004, 41 (22-23), 6111-6127.

(66) Diani, J. Int. J. Fract. 2001, 112 (2), 151-161.

(67) Biwa, S. Int. J. Nonlinear Mech. 2006, 41 (9), 1084-1094.

(68) Williams, M. L.; Schapery, R. A. Int. J. Fracture Mech. 1965, 1 (1), 64-71.

(69) Lazzeri, A.; Bucknall, C. B. J. Mater. Sci. 1993, 28 (24), 67996808.

(70) Fond, C.; Lobbrecht, A.; Schirrer, R. Int. J. Fract. 1996, 77, 141159.

(71) Long, R.; Hui, C. Y. Soft Matter 2010, 6 (6), 1238-1245.

(72) Zebarjad, S. M.; Bagheri, R.; Lazzeri, A.; Serajzadeh, S. Mater. Design 2004, 25 (3), 247-250.

(73) Wu, W. J. Polym. Sci., Polym. Phys. 1980, 18 (7), 1659-1661. 